ARTICLE

\title{
Eltrombopag directly inhibits BAX and prevents cell death
}

\author{
Adam Z. Spitz ${ }^{1,2}$, Emmanouil Zacharioudakis ${ }^{1,2}$, Denis E. Reyna ${ }^{1,2}$, Thomas P. Garner ${ }^{1,2}$ \& \\ Evripidis Gavathiotis (10 1,2,3,4,5凶
}

The BCL-2 family protein BAX has essential activity in mitochondrial regulation of cell death. While BAX activity ensures tissue homeostasis, when dysregulated it contributes to aberrant cell death in several diseases. During cellular stress BAX is transformed from an inactive cytosolic conformation to a toxic mitochondrial oligomer. Although the BAX transformation process is not well understood, drugs that interfere with this process are useful research tools and potential therapeutics. Here, we show that Eltrombopag, an FDA-approved drug, is a direct inhibitor of BAX. Eltrombopag binds the BAX trigger site distinctly from BAX activators, preventing them from triggering $B A X$ conformational transformation and simultaneously promoting stabilization of the inactive BAX structure. Accordingly, Eltrombopag is capable of inhibiting BAX-mediated apoptosis induced by cytotoxic stimuli. Our data demonstrate structure-function insights into a mechanism of BAX inhibition and reveal a mechanism for Eltrombopag that may expand its use in diseases of uncontrolled cell death.

\footnotetext{
${ }^{1}$ Department of Biochemistry, Albert Einstein College of Medicine, Bronx, NY, USA. ${ }^{2}$ Department of Medicine, Albert Einstein College of Medicine, Bronx, NY, USA. ${ }^{3}$ Albert Einstein Cancer Center, Albert Einstein College of Medicine, Bronx, NY, USA. ${ }^{4}$ Wilf Family Cardiovascular Research Institute, Albert Einstein College of Medicine, Bronx, NY, USA. ${ }^{5}$ Institute of Aging Research, Albert Einstein College of Medicine, Bronx, NY, USA.

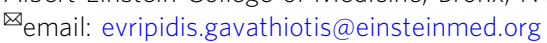


CL-2 family proteins are principal regulators of apoptosis in health and diseases ${ }^{1-4}$. Mitochondrial outer membrane permeabilization (MOMP) is a key event that defines apoptotic cell death ${ }^{5,6}$. MOMP releases apoptogenic factors such as cytochrome $c$ into the cytosol, which in turn irreversibly execute the apoptotic signaling cascade ${ }^{5,6}$. Pro-apoptotic BCL-2 proteins BAX and BAK play a key role in this process due to their ability to transform into mitochondrial outer membraneembedded oligomers that induce $\mathrm{MOMP}^{7,8}$. In cells, BAX and BAK can exist as an inactive monomer, autoinhibited homodimer, or a neutralized conformation bound to anti-apoptotic BCL-2 family members such as BCL-2, BCL-xL, and MCL- ${ }^{9-13}$. The pro-apoptotic "BH3-only" proteins such as BIM, BID, and PUMA, which comprise the third class of the BCL-2 family, sense cellular stress and utilize their BCL-2 homology 3 (BH3) domain helix to either neutralize the anti-apoptotic BCL-2 proteins and/ or directly activate pro-apoptotic BAX and BAK and initiate their conformational transformation ${ }^{13-15}$.

$\mathrm{BAX}$ activation is a dynamic process that occurs upon binding of a $\mathrm{BH} 3$-only protein with its $\mathrm{BH} 3$ domain helix to the $\mathrm{N}$ - terminal BAX trigger site ( $\alpha 1, \alpha 6$ helices), inducing several conformational changes ${ }^{16-22}$ (Fig. 1a, b). The release of the helix $\alpha 9$ from the C-terminal canonical site of BAX, formed by $\alpha 3-\alpha 5$ helices, allows BAX to translocate from the cytosol and insert into the mitochondrial outer membrane (MOM) (Fig. 1c) ${ }^{18-21}$. Once translocated, BAX forms homo-oligomeric pores which permeabilize the $\mathrm{MOM}^{20-22}$.

While BAX-mediated cell death contributes to tissue homeostasis and killing of malfunctioning cells, genetic deletion of BAX alone has shown successful protection from excess cell death in various disease models ${ }^{23-27}$. Small molecules that can directly modulate $\mathrm{BAX}$ can be useful probes to investigate the role of BAX in the context of various biological mechanisms and disease models ${ }^{28,29}$. Such chemical probes can aid in identifying BAX regulatory sites and elucidate the complex conformational transformation of BAX. Indeed, we previously identified smallmolecule BAX inhibitors that bind an allosteric binding site on inactive BAX, which is distinct from previously known regulatory sites ${ }^{30}$. These small molecules trap BAX in an inactive state and have shown efficacy in preventing cardiomyocyte cell

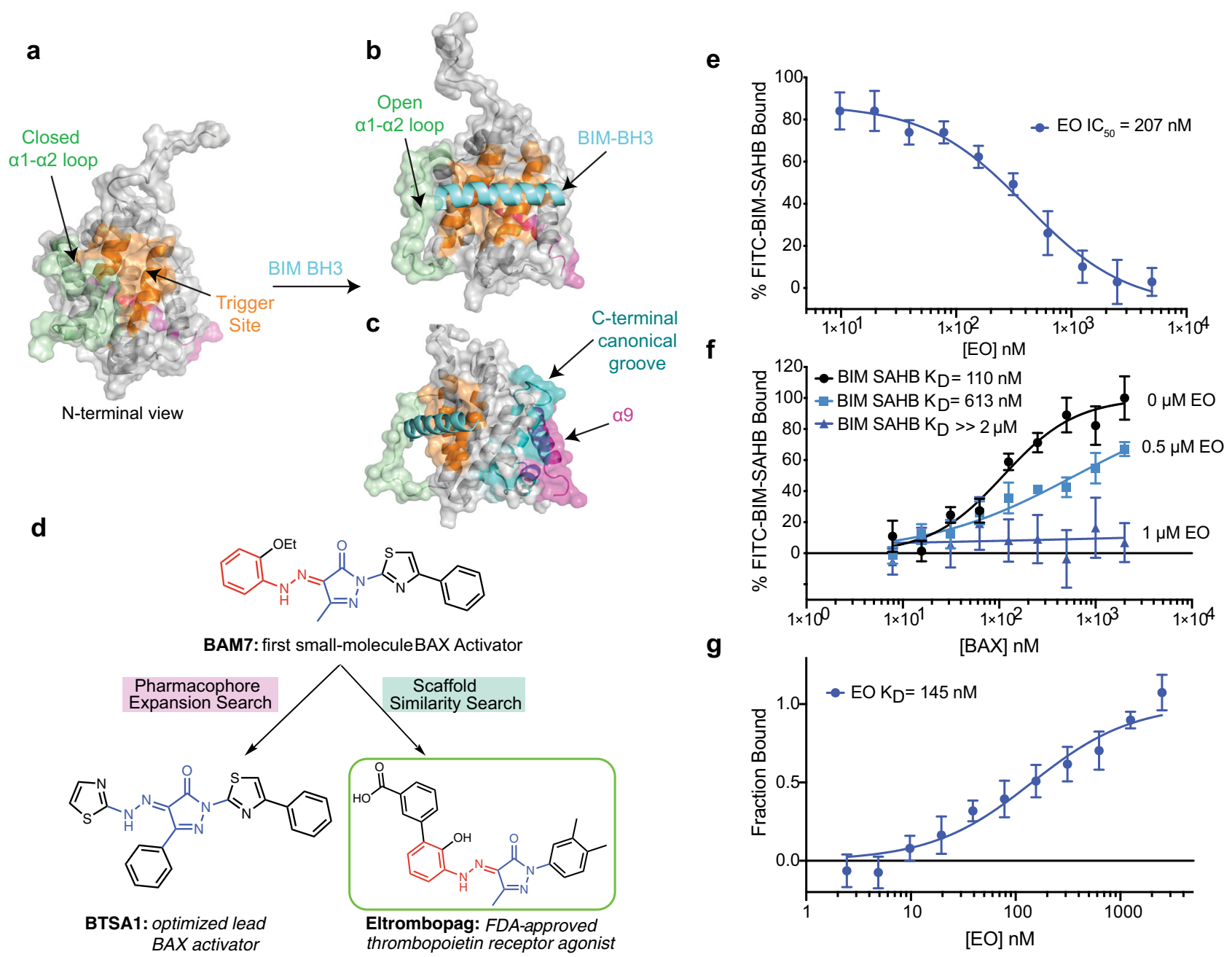

Fig. 1 Eltrombopag potently binds BAX. a Surface representation of the inactive BAX structure (PDB 1F16: https://doi.org/10.2210/pdb1F16/pdb) showing the location of the BAX trigger site (orange) and closed $\alpha 1-\alpha 2$ loop (green). b, c Surface representation of the BIM-BH3-bound active conformation (PDB 2K7W: https://doi.org/10.2210/pdb2K7W/pdb) showing binding of BIM-BH3 (cyan) with opened $\alpha 1-\alpha 2$ loop (green) at the trigger site (b) and the location of $\alpha 9$ bound to the C-terminal canonical groove (c). d Chemical structures of BAM7, BTSA1, and EO derived by similarity search. The 3-methyl pyrazolone (blue) and phenylhydrazone (red) groups are highlighted for clarity. e Competitive fluorescence polarization (FP) binding assay of EO. Data are representative of three independent experiments, each $n=3 \pm$ SEM. $\mathbf{f}$ Binding affinity of FITC-BIM-SAHB to BAX by FP in the presence of EO. Data represent $n=2 \pm$ SEM from two independent experiments. $\mathbf{g}$ Microscale thermophoresis direct binding of EO to BAX-4C. Data are representative of three independent experiments each $n=3 \pm$ SEM. Source data for this figure is provided. 
death in pre-clinical animal models of doxorubicin-induced cardiomyopathy ${ }^{31}$.

The discovery of novel BAX targeting small molecules offers an opportunity to develop them into drugs. However, repurposing of clinically approved small molecules is a highly attractive goal given the tremendous challenges of de-novo drug discovery and development ${ }^{32}$. While searching for direct BAX modulators based on the previous small-molecule BAX activators ${ }^{33,34}$, we identified Eltrombopag (EO), an FDA-approved thrombopoietin receptor agonist and iron chelator that is used to increase blood platelet counts due to chronic immune thrombocytopenia ${ }^{35,36}$. Despite some similarity with the BAX activators, EO proved instead to be a direct inhibitor of BAX. Thus, we characterized the mechanism of BAX inhibition providing structural and functional insights and investigated whether EO can target BAX intracellularly and inhibit BAX-mediated cell death.

\section{Results}

Eltrombopag binds to BAX. To identify small-molecule BAX modulators, we explored pharmacophore enhancement and substructure similarity computational methods based on the small-molecule activator of BAX, BAM7; $\mathbf{1}^{33}$. Searching a library of FDA-approved small molecules using the 3-methyl pyrazolone and phenylhydrazone core as a query, we identified as a hit, Eltrombopag (EO; 2) (Fig. 1d). EO bears a striking similarity to BAM7, sharing the 3-methyl pyrazolone and phenylhydrazone core, however, substitutions at either side to this core with dimethylphenyl and benzoic acid markedly distinguish EO from BAM7 and the optimized lead BAX activator, BTSA1, $3^{33,34}$ (Fig. 1d).

We hypothesized EO would exhibit some binding interaction with the N-terminal BAX trigger site. We therefore used a competitive fluorescence polarization assay (FPA) ${ }^{33}$ based on the interaction between recombinant BAX and fluorescently-labeled stapled BIM-BH3 peptide (FITC-BIM-SAHB) ${ }^{17}$. EO dosedependently competed FITC-BIM-SAHB with a remarkable half-maximal inhibition $\mathrm{IC}_{50}$ of $207 \mathrm{nM}$ (Fig. 1e). Titrations of BAX to a constant concentration FITC-BIM-SAHB exhibited a decreased affinity in the presence of constant concentrations of EO (Fig. 1f). Direct binding of EO to BAX was demonstrated using microscale thermophoresis (MST) with a calculated dissociation constant $\mathrm{K}_{\mathrm{D}}$ of $143 \mathrm{nM}$ (Fig. $1 \mathrm{~g}$ and Supplementary Fig. 1a, b). The $\mathrm{IC}_{50}$ and $\mathrm{K}_{\mathrm{D}}$ are consistent with a competitive binding mechanism where EO directly displaces FITCBIM-SAHB from the N-terminal trigger site of BAX.

Eltrombopag inhibits BAX activation. We evaluated EO's capacity to modulate BAX activity using liposomal release assays. EO exhibited no ability to activate recombinant BAX in concentrations even up to $10 \mu \mathrm{M}$ (Fig. 2a). Instead, EO was able to inhibit both tBID- and BIM-BH3-mediated BAX activation (Fig. 2b-d and Supplementary Fig. 2a-c). Based on the competitive nature of EO binding to the $\mathrm{N}$-terminal BAX trigger site, we predicted that EO inhibition of BAX activity would be dependent on the $\mathrm{BH} 3$-only activator concentration. Indeed, inhibition of tBID-mediated BAX activation by EO was inversely proportional to the concentration of the tBID activator, consistent with competitive inhibition of $\mathrm{BH} 3$-activator binding (Supplementary Fig. 2d-f). Furthermore, EO was capable of inhibiting heatinduced BAX activation, suggesting that $\mathrm{EO}$ can stabilize inactive BAX in addition to blocking the BAX activation site from BH3mediated activators (Fig. 2e and Supplementary Fig. 3a-c). EO exhibited similar low micromolar potency in inhibiting BAX activity in liposomal release assay against all stimuli $\left(\mathrm{tBID} \mathrm{IC}_{50}=\right.$
$2.4 \mu \mathrm{M}, \mathrm{BIM}-\mathrm{BH} 3 \mathrm{IC}_{50}=4.7 \mu \mathrm{M}$, heat $\mathrm{IC}_{50}=4.5 \mu \mathrm{M}$ ). (Fig. $2 \mathrm{f}$ and Supplementary Fig. 3c).

Prior to permeabilizing membranes, activated BAX must first translocate to the membrane ${ }^{37}$. To explore this earlier step in BAX activation, we utilized an NBD-fluorescence based translocation assay (Fig. $2 \mathrm{~g}-\mathrm{i}$ and Supplementary Fig. 3d, e). EO was capable of inhibiting tBID- and BIM-BH3-induced BAX translocation with comparable potency to that of inhibiting liposomal release $\left(\mathrm{tBID} \mathrm{IC}_{50}=6.3 \mu \mathrm{M}, \mathrm{BIM}-\mathrm{BH} 3 \quad \mathrm{IC}_{50}=5.7\right.$ $\mu \mathrm{M})$. Similarly, EO was capable of inhibiting heat-induced autotranslocation of BAX, suggesting that EO binding stabilizes a soluble conformation of BAX (Supplementary Fig. 3f, g). One of the earliest conformational changes of BH3-mediated BAX activation is the opening of the $\alpha 1-\alpha 2$ loop and the exposure of an N-terminal 6A7 epitope ${ }^{16,18}$. In an immunoprecipitation assay using the anti-6A7 epitope-specific antibody, BIM-BH3-induced 6A7 exposure as previously shown, but it was inhibited by the presence of EO (Supplementary Fig. 3h). Taken together, the data suggest $\mathrm{EO}$ inhibits $\mathrm{BAX}$ activation process at an early stage by blocking activator binding and stabilizing a soluble inactive form of BAX.

Eltrombopag forms unique contacts at the BAX trigger site. While a high-resolution structure of the BAX-EO complex would be ideal, such structures have been elusive due to technical challenges resulting from the dynamic BAX structure. To determine the binding site of EO, we performed $2 \mathrm{D}{ }^{1} \mathrm{H}-{ }^{15} \mathrm{~N}$ heteronuclear single quantum coherence (HSQC) NMR analysis with ${ }^{15} \mathrm{~N}$-labeled BAX. EO titration shifted select cross-peaks of corresponding BAX residues in the NMR spectra. Analysis of the chemical shift perturbations (CSPs) of BAX in the presence of EO indicated small and specific shifts, as with previous NMR studies of $\mathrm{BAX}^{17,18,33,34,38,39}$. Significant CSPs were localized predominantly to the $\mathrm{N}$-terminal BAX trigger site, specifically the N-terminal region of $\alpha 1$ and the length of $\alpha 6$ (Fig. 3a). Mapping of the CSPs onto the inactive BAX structure revealed that CSPs localizing to the BAX trigger site coalesce to form a contiguous surface with a shallow hydrophobic pocket between $\alpha 1$ and $a 6$ (Fig. 3b). Additional CSPs corresponding to residues in adjacent helices to the trigger site, $\alpha 4$ and $\alpha 7$ but also distant at the C-terminal $\alpha 9$ were observed (Fig. 3a, b). Previous crystal structures of the inactive BAX mutants P168G and W139A suggested that binding at the $\mathrm{N}$-terminal trigger site may modulate BAX activity via local conformational changes at $\alpha 4$, $\alpha 7$, and $\alpha 9^{40}$. NMR analysis of BAX activation with BIM-SAHB and small-molecule trigger site activators also highlighted allosteric sensing in $\alpha 4, \alpha 7$, and $\alpha 9^{17,33}$. The observed distal CSPs could correspond to similar conformational effects from the binding of EO to the BAX trigger site. Notably, we observed few chemical shift perturbations in N-terminal a1-a2 loop residues, suggesting that its structure remains largely unchanged upon EO binding (Fig. 3a, b). This is in direct contrast with BIM-SAHB and BTSA1 binding, which induce significant CSPs in $\alpha 1-\alpha 2$ loop residues and corresponding with the displacement of the $a 1-\alpha 2$ loop from the trigger site, a critical step in the activation of $\mathrm{BAX}^{17,33,34}$.

We next performed CSP-guided molecular docking of EO to the BAX surface to determine the binding pose of $\mathrm{EO}$ to the inactive BAX structure (PDB: 1F16: https://doi.org/10.2210/ pdb1F16/pd $)^{16}$. We predicted that the negatively charged EO carboxylate would form a favorable interaction with one of the three basic residues at the EO-binding interface highlighted by the NMR data, K21, R134, or R145, all of which exhibited significant CSPs (Fig. 3a). We, therefore, performed molecular docking of EO to a site centered about residues K21, R134, and 
a

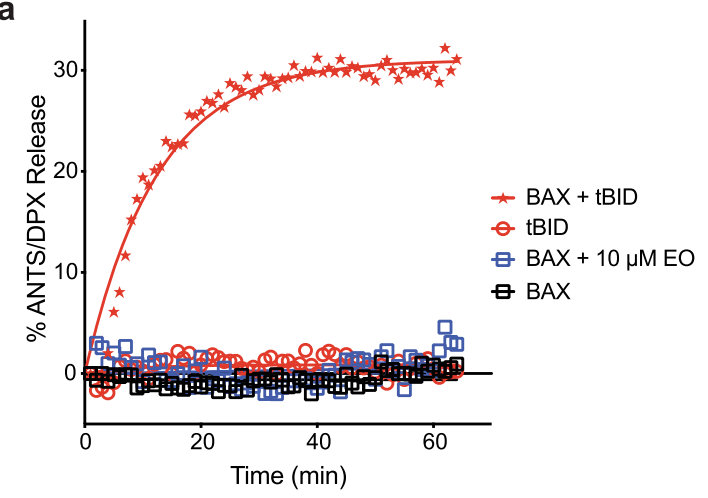

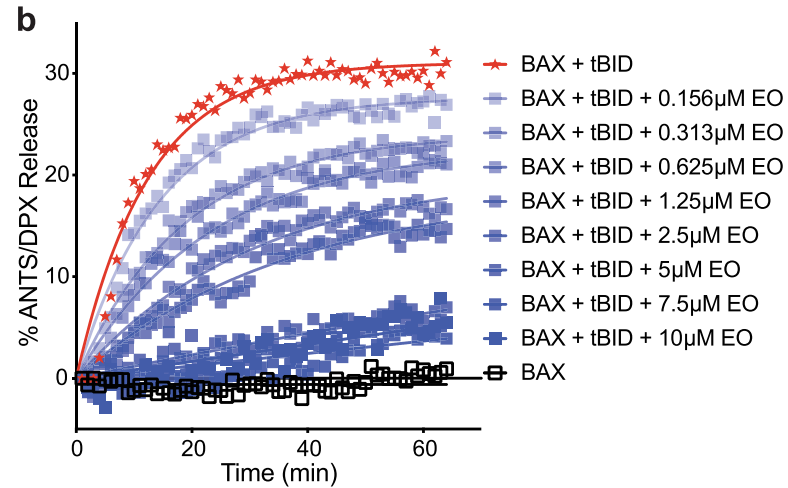

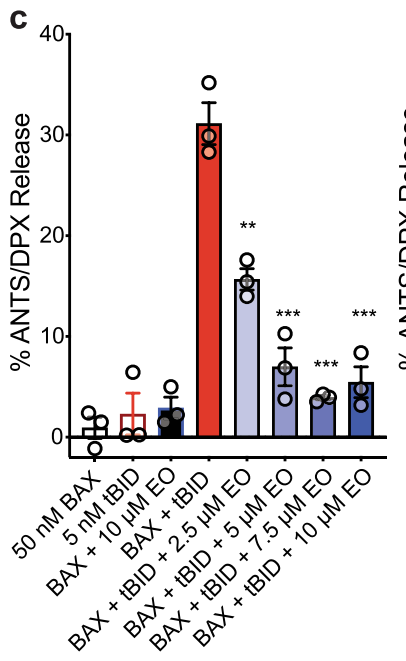

e
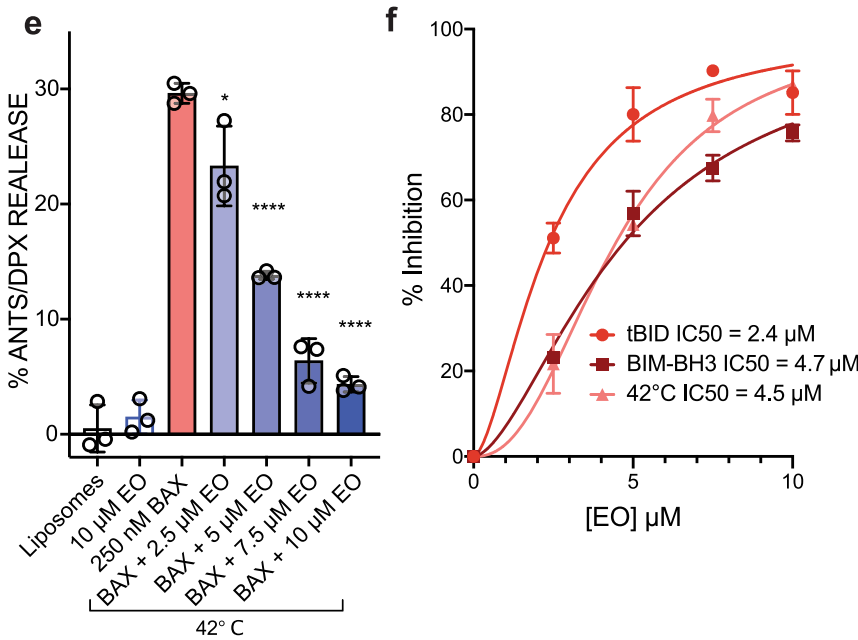

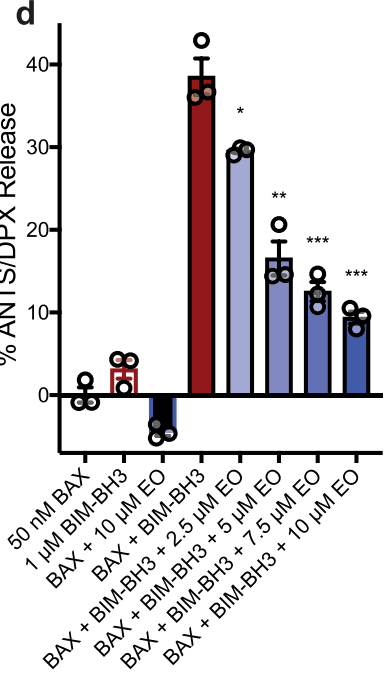

$h$

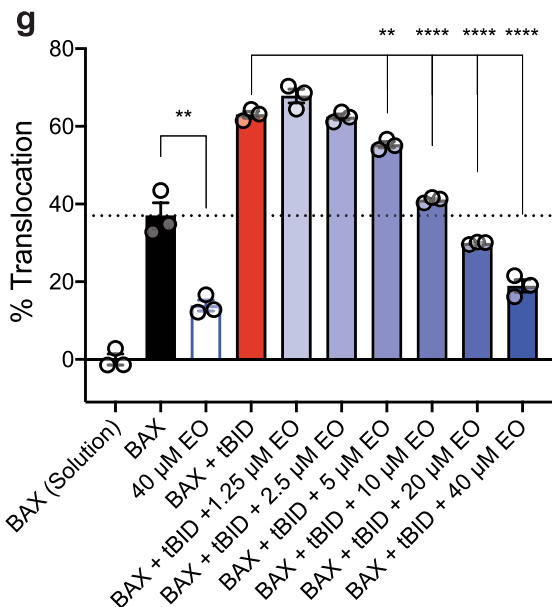

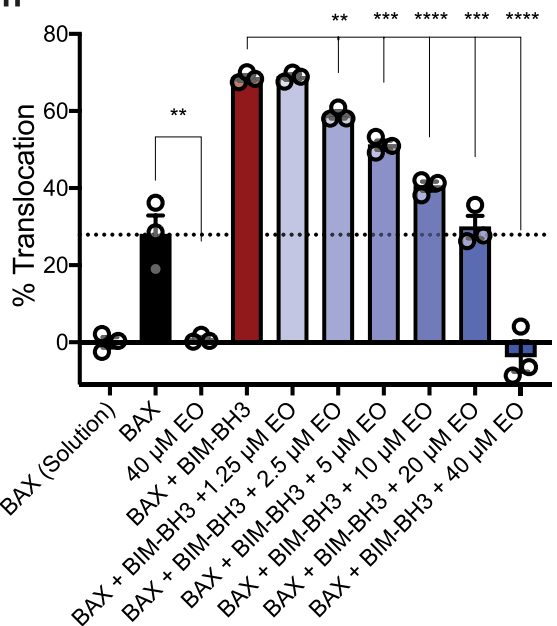

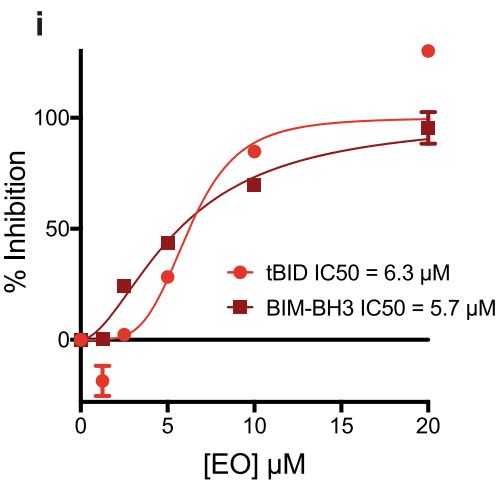

Fig. 2 Eltrombopag inhibits BAX activation. a-e BAX-mediated membrane permeabilization assay using liposomes with $50 \mathrm{nM} B A X$ and $5 \mathrm{nM}$ tBID (a-c), $50 \mathrm{nM} \mathrm{BAX}$ and $1 \mu \mathrm{M} \mathrm{BIM-BH3}(\mathbf{d})$, or $250 \mathrm{nM} \mathrm{BAX}$ at $42{ }^{\circ} \mathrm{C}(\mathbf{e})$, each at $30 \mathrm{~min}$. Data are representative of three independent experiments, each $n=3 \pm$ SEM. $\mathbf{f}$ Summary percentage inhibition curve for all liposomal release stimuli with $I C_{50}$ included for clarity. Data are representative of three independent experiments, each $n=3 \pm$ SEM. $\mathbf{g}$, $\mathbf{h}$ Membrane translocation assay using NBD-labeled BAX ( $800 \mathrm{nM}$ ) activated by tBID (200 nM) (g) and BIM-BH3 (1 $\mu \mathrm{M})(\mathbf{h})$ each at $120 \mathrm{~min}$. Data are representative of three independent experiments, each $n=3 \pm$ SEM. i Summary percentage inhibition curve for all BAX translocation stimuli with $I C_{50}$ included for clarity. Data are representative of three independent experiments, each $n=3 \pm$ SEM. Two-sided $t$ test, ${ }^{\star \star \star \star *} P<$ $0.0001 ;{ }^{\star \star \star} P<0.001 ;{ }^{\star \star} P<0.01 ;{ }^{\star} P<0.05 ; \mathrm{ns}, P>0.05$. (left to right $P$ values were $0.0027,0.0010,0.0002,0.0006$ for (c), 0.0154, 0.0019, 0.0005, 0.0002 for (d), $0.0379,0.000008,0.00004,0.000002$ for $(\mathbf{e}), 0.0029,0.0025,0.00002,0.000003,0.00002$ for $(\mathbf{g}), 0.0056,0.0014,0.0002$, $0.00004,0.0002,0.00006$ for $(\mathbf{h}))$. Source data for this figure is provided.

R145. We performed induced-fit docking (IFD) with Schrödinger software using a largely extended surface of BAX at the trigger site to account for potential ambiguity with the NMR data and exhaustively consider possible binding modes and the associated local conformational changes of $\alpha 1, \alpha 6$, and $\alpha 1-\alpha 2$ loop residues. The IFD yielded several poses which featured ionic interactions between the EO carboxylate and K21, R134, or R145 as expected (Supplementary Fig. 4a, b). 


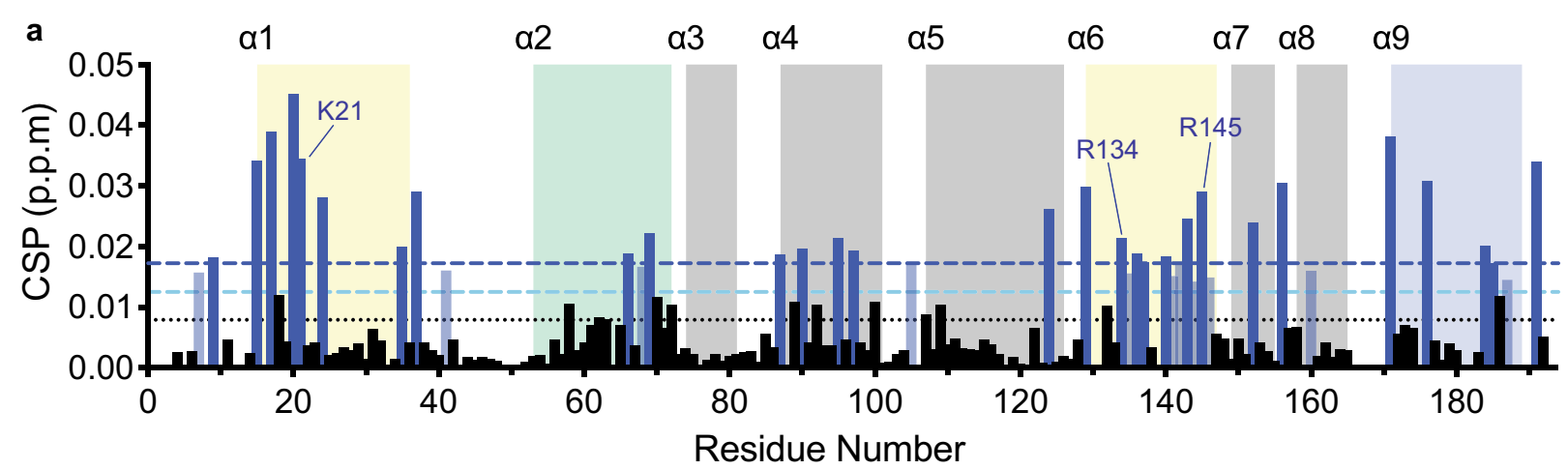

b
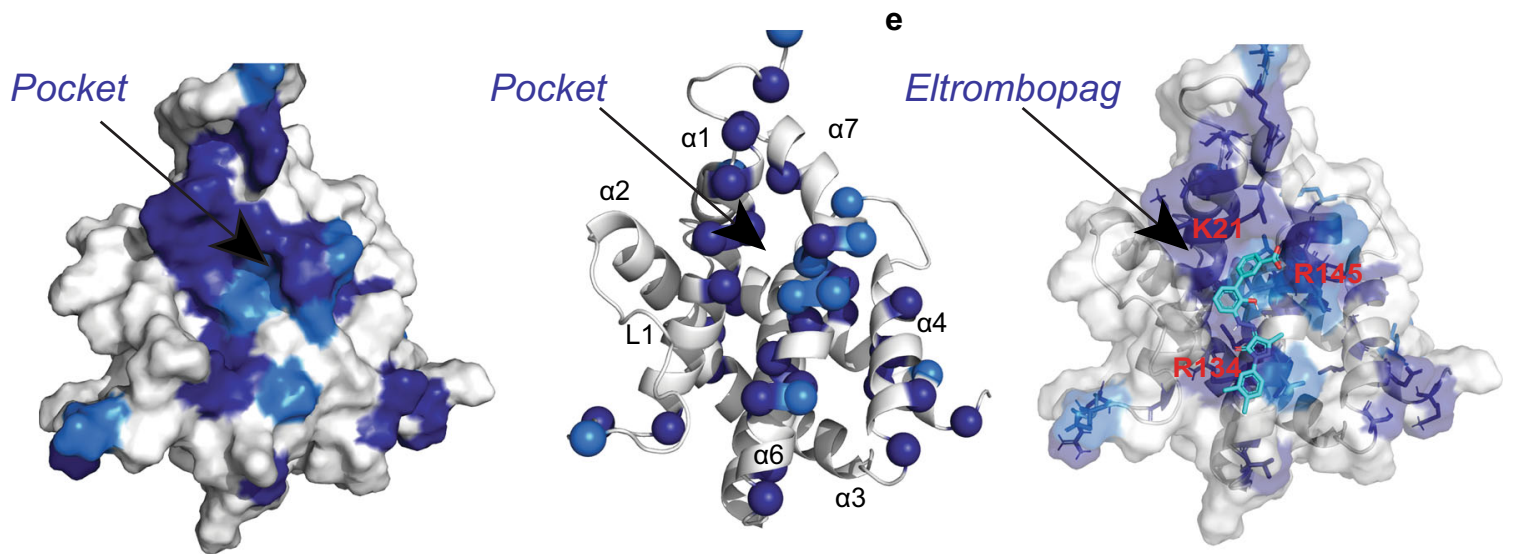

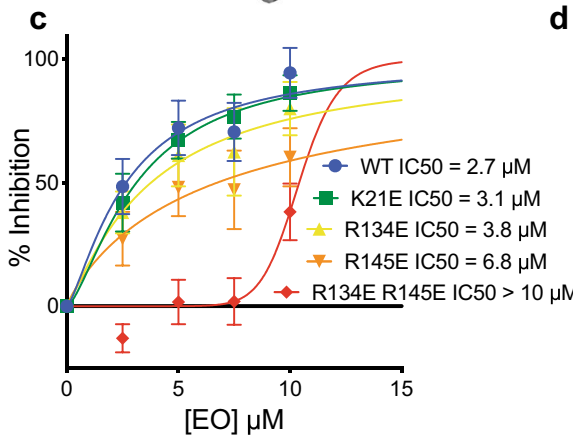

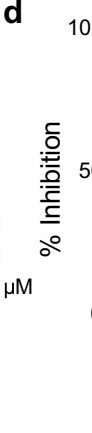

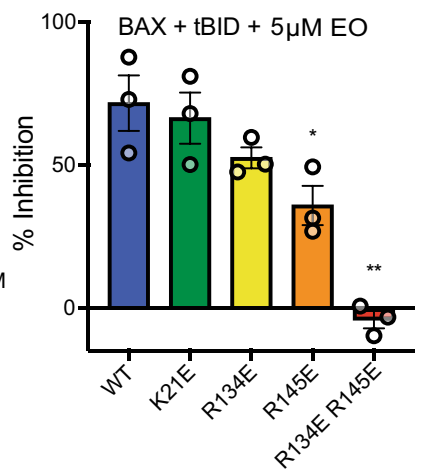

h

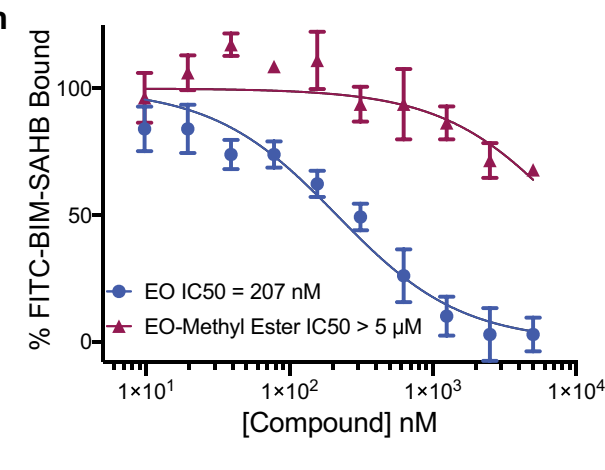

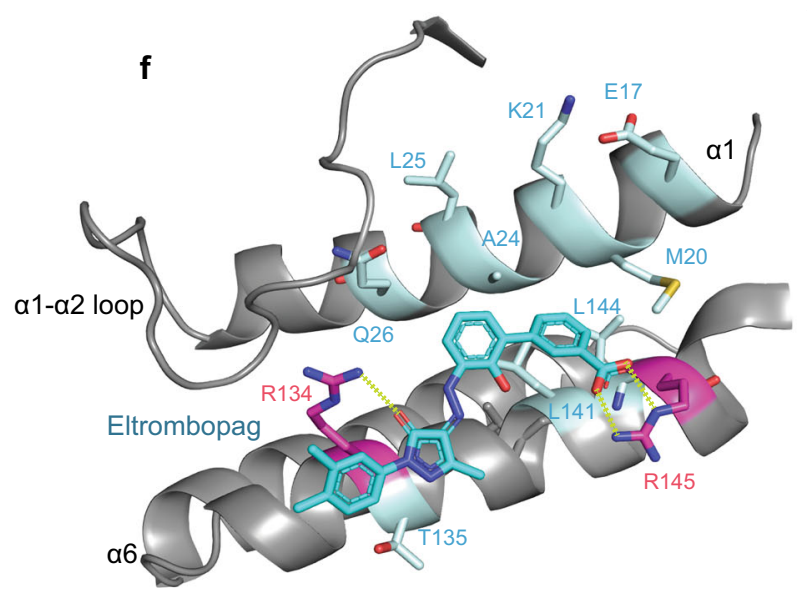

g

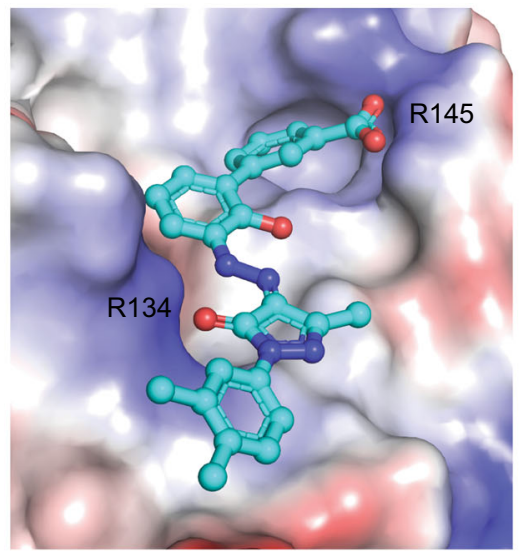

We set out to determine which EO pose was appropriate by comparing BAX trigger site mutants that would eliminate one of the three basic trigger site residues, K21E, R134E, or R145E, to wild-type (WT). All tested mutants exhibited identical retention time in size-exclusion chromatography analysis, suggesting no effect on BAX folding and had comparable purity and molecular weight to WT BAX as determined by SDS-PAGE (Supplementary
Fig. 4c, d). In liposomal release assays, all of the BAX mutants were functional, although R134E and R145E exhibited lower ANTS/DPX release in response to tBID activation (Supplementary Fig. 4e). Of the mutants tested, only BAX R145E exhibited a reduced inhibition in response to $\mathrm{EO}$, with an $\mathrm{IC}_{50}$ more than double that of BAX WT (Fig. 3c, d). Consistently, HSQC-NMR analysis of ${ }^{15} \mathrm{~N}$-labeled BAX R145E in the presence of EO 
Fig. 3 Eltrombopag binds the BAX trigger using unique contacts. a Measured chemical shift perturbations (CSPs) of ${ }^{15} \mathrm{~N}$-labeled $\mathrm{BAX}$ in the presence of 1:2 BAX:EO are plotted as a function of BAX residue number. Residues with chemical shift perturbations over the significance threshold or two times the significance threshold are labeled light blue or dark blue, respectively. The black dotted line represents the average CSP. Residues associated with the Nterminal trigger site, $\mathrm{BH} 3$ domain, canonical site, and transmembrane domain are highlighted in yellow, green, gray, and blue, respectively. Basic residues of the N-terminal trigger site are labeled for clarity. Data are representative of three independent experiments. $\mathbf{b}$ Mapping of residues undergoing significant CSPs to the surface and the ribbon structure of BAX (PDB: 1F16: https://doi.org/10.2210/pdb1F16/pdb). Residues with significant CSPs cluster on the Nterminal trigger site of BAX surrounding a hydrophobic pocket formed by $\alpha 1$ and $\alpha 6$. $\mathbf{c}, \mathbf{d}$ Percent inhibition of BAX-mediated membrane permeabilization assay using liposomes with $250 \mathrm{nM} \mathrm{BAX}$ and $5 \mathrm{nM}$ tBID with various trigger site mutants, dose-response IC 50 (c) and bar graph for $5 \mu \mathrm{M}$ EO (d) are shown for clarity. Data are representative of two independent experiments, each $n=3 \pm$ SEM. e Transparent surface with ribbon representation of the EO binding site as determined by NMR data and docking. $\mathbf{f}$ Close up view of the EO-binding site with residues determined by NMR data forming hydrophobic contacts with EO are highlighted in cyan and residues forming specific interactions, R134 and R145, are highlighted in red. $\mathbf{g}$ BAX electrostatic surface representation highlighting positive (blue) and negative (red) charge as a gradient and hydrophobic surfaces as gray. $\mathbf{h}$ Competitive fluorescence polarization binding assay of EO and inactive EO-methyl ester analog. Data are representative of three independent experiments, $n=3 \pm \mathrm{SEM}$. Two-sided $t$ test, ${ }^{\star \star \star \star} P<0.0001$; ${ }^{\star \star \star} P<0.001 ;{ }^{\star \star} P<0.01 ;{ }^{\star} P<0.05 ; \mathrm{ns}, P>0.05$. (left to right $P$ values were $0.0395,0.0017$ for $(\mathbf{d})$ ). Source data for this figure is provided.

exhibited few non-localized CSPs, indicating a significant reduction in EO-BAX affinity (Supplementary Fig. 4f, g). Notably, BAX K21E exhibits reduced activation in response to BIM-BH3, BAM7, and BTSA1 activators but not reduced inhibition in response to $\mathrm{EO}^{17,18,33,34}$. This highlights how unique contacts at the trigger site could determine whether compounds will behave as BAX activators or inhibitors.

The loss of EO-mediated BAX inhibition with the R145E mutant suggests that EO forms a critical interaction via the anionic carboxylate with BAX R145. With this known, we reevaluated the EO docking poses and analyzed the top pose featuring an ionic interaction between the EO carboxylate and the side chain of R145 (Fig. 3e-g). In addition to the ionic interaction, this pose also features hydrophobic interactions between the biphenyl moiety of EO and the hydrophobic pocket formed by residues L24, M137, G138, and L141 between a1 and a6. Furthermore, the docking pose features contacts at the $\mathrm{N}$ terminal of $a 6$ unique to poses possessing an ionic interaction with R145 (Supplementary Fig. 4a, b). Computational prediction of BAX-binding energy for EO, BAM7, and BTSA1 accurately predicted this pose of EO to be the highest affinity and correlated to the observed IC50 values in FPA (Supplementary Fig. 4h). Of particular note is a hydrogen bond between the R134 side chain and the carbonyl of the pyrazolone core of EO, which could potentially explain the slight trend toward weaker inhibition of BAX R134E (Fig. 3c, d). HSQC-NMR analysis of ${ }^{15} \mathrm{~N}$-labeled BAX R134E in the presence of EO exhibited clustering of CSPs to the trigger site, albeit with reduced CSPs compared to WT BAX (Supplementary Fig. $4 \mathrm{i}-\mathrm{j}$ ). We, therefore, hypothesized that the double-mutant BAX R134E R145E would markedly reduce EO inhibition of BAX. Indeed, EO exhibited clearly weak inhibition of BAX R134E R145E with an $\mathrm{IC}_{50}>10 \mu \mathrm{M}$ (Fig. 3c, d). Consistently, in the presence of EO, this double mutant also exhibited minimal CSPs of ${ }^{15} \mathrm{~N}$-labeled BAX by HSQC-NMR studies and markedly reduced affinity as determined by MST (Supplementary Fig. 5a-c and Fig. 1a, c).

To further probe the specificity of EO for the BAX trigger site and the critical R145 ionic interaction, we utilized an EO analog featuring a methyl ester (EO-Methyl Ester; 4) in place of the carboxylic acid (Supplementary Fig. 5d). The addition of a methyl group eliminates the anionic carboxylate as well as adds steric bulk at the site of the critical R145 interaction (Fig. 3e-g). EOMethyl Ester exhibited minimal competition of FITC-BIM-SAHB by FPA $\left(\mathrm{IC}_{50}>5 \mu \mathrm{M}\right)$, indicating a dramatically diminished binding affinity (Fig. 3h). Consistently, EO-Methyl Ester induced minimal CSPs of ${ }^{15} \mathrm{~N}$-labeled BAX by HSQC-NMR studies and exhibited significantly diminished inhibition of BAX in liposomal release assays (Supplementary Fig. 5e-h). Taken together, the data show that EO binds to the N-terminal trigger site of BAX forming contacts with a shallow hydrophobic pocket between a1 and $\alpha 6$, interacting predominantly with $\alpha 6$ residues. Notably, this pocket is formed adjacent to the $\alpha 1-\alpha 2$ loop, which interacts with residues of $\alpha 1$ and $a 6$ and it is not disturbed by EO binding. Docking, mutagenesis, and the EO-Methyl Ester suggest EO makes a critical contact with R145 via the anionic carboxylate as well as a secondary hydrogen bond with R134 via the pyrazolone carbonyl.

Stabilization of inactive BAX by Eltrombopag. Our data suggest that the N-terminal BAX trigger site, which has been established as the binding site for both BH3-only proteins and smallmolecule activators, could also be an inhibitory site. NMR and mutagenesis data indicated that the distinct contacts and binding mode of EO may be responsible for the inhibitory activity of EO. To explore how EO can accomplish inhibition of BAX conformation and activity, we performed three independent molecular dynamics (MD) simulations of the BAX-EO complex and of the inactive BAX structure. The overall structure of BAX was maintained in all six simulations as determined by similar RMSD and radius of gyration in the presence and absence of EO (Supplementary Fig. $6 \mathrm{a}-\mathrm{h})$. In the three MD simulations of the BAXEO complex, EO remains in a stable conformation (Fig. 4a and Supplementary Fig. 6a-f). The distance between R145 and the EO carboxylate remains stable throughout the simulation (Fig. 4b). The interaction between R134 and the EO carbonyl is noticeably more dynamic, however the two groups remain in close proximity throughout the simulation (Fig. 4c). The EO-BAX distances strongly support the mutagenesis data and binding mode wherein EO forms a critical interaction at R145 and a secondary weaker interaction at R134.

HSQC CSPs suggested that EO does not cause significant conformational changes to $\alpha 1-\alpha 2$ loop, in contrast with other trigger site binders, BTSA1 and BH3 peptides ${ }^{33,34,41}$. In the unbound BAX structure, R134 on $a 6$ sits in close proximity to E44 and D48 on $\alpha 1-\alpha 2$ loop $^{16}$. The distance between R134 and D48 is approximately equal for both the BAX and BAX-EO simulations (Fig. 4d and Supplementary Fig. 7a, b). However, R134 and E44 remain in closer proximity in simulations of the BAX-EO complex than in BAX alone (Fig. 4e and Supplementary Fig. 7c, d). Furthermore, the BAX-EO simulations displayed a narrower distribution of distances indicating reduced conformational flexibility in the N-terminal region of $\alpha 1-\alpha 2$ loop. Notably, the few CSPs observed on $\alpha 1-\alpha 2$ loop were towards the $\mathrm{N}$ terminal region (Fig. 3a).

To further explore potential conformational changes associated with EO binding to BAX, we analyzed the percentage change in root-mean-square fluctuation (RMSF), a measure of the dynamics of each residue in the BAX structure (Fig. $4 \mathrm{f}$ and Supplementary 
d

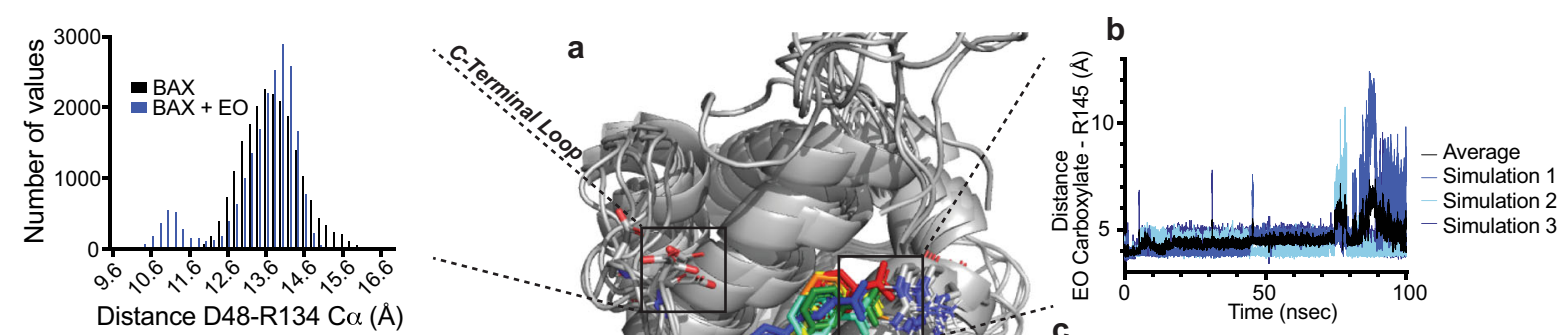

e

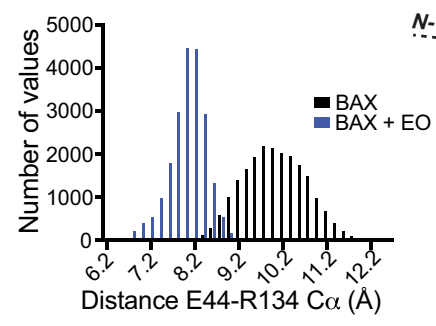

istance E44-R134 $\mathrm{C} \alpha(\AA)$

f

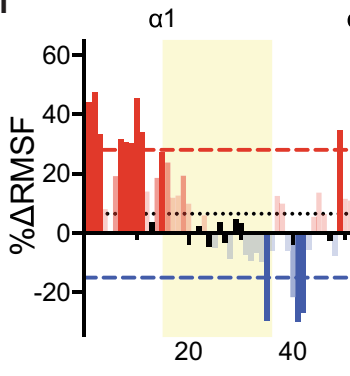

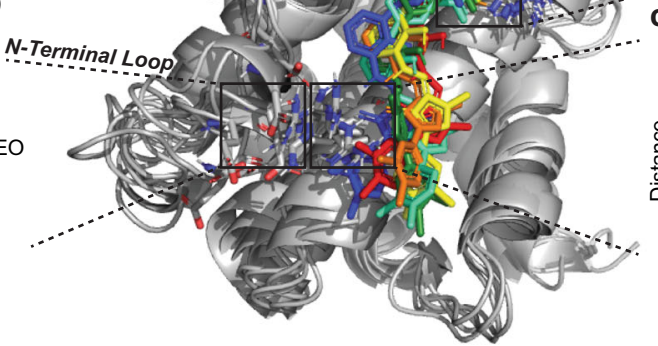

c

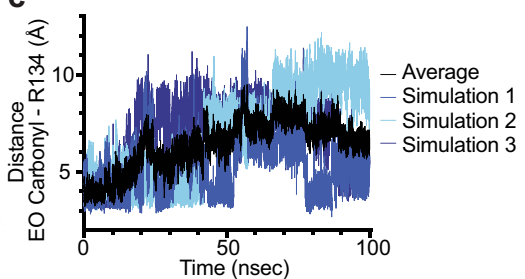

g

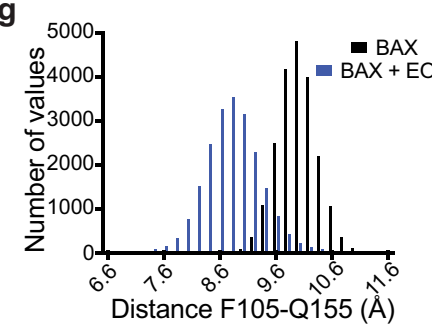

h

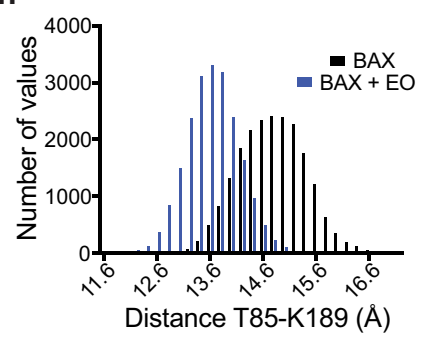

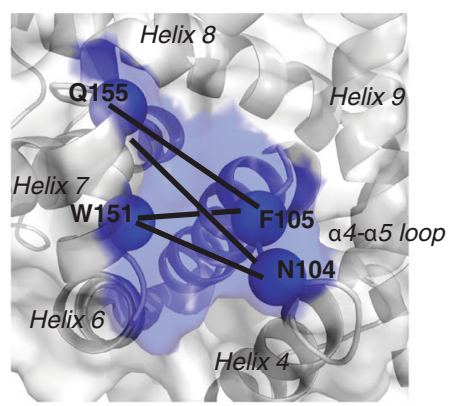

$\begin{array}{lll}80 & 100 \quad 120\end{array}$

Residue Number $\begin{array}{lll}\alpha 7 & \alpha 8 & \alpha 9\end{array}$

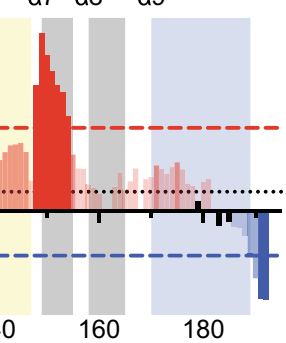

| $>25 \%$

| $15 \%-25 \%$

$5 \%-15 \%$

I $-5 \%-5 \%$

$-5 \%--15 \%$

| $-15 \%--25 \%$

| $<-25 \%$
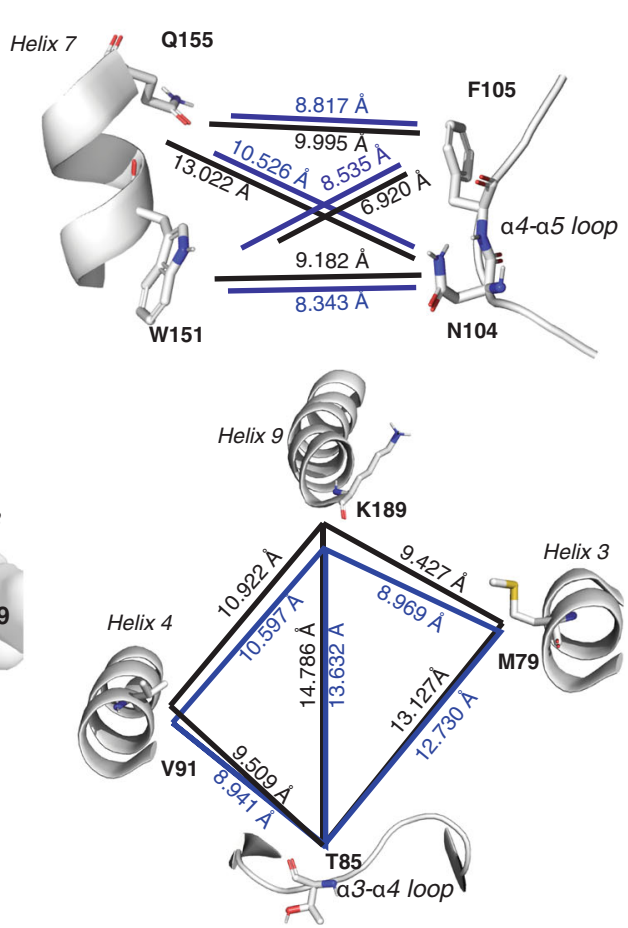

Fig. 8). Although no net change in RMSD was observed, discrete clusters of residues exhibited increases and decreases in dynamics. Residues towards the $\mathrm{N}$ and $\mathrm{C}$-terminus of $\alpha 1-\alpha 2$ loop exhibit reduced and increased RMSF, respectively, as expected based on a1- $\alpha 2$ loop distances to R134. Two additional regions exhibited dramatic changes in RMSF. The $\alpha 4-\alpha 5$ loop and helix $\alpha 7$ exhibit an increase in RMSF whereas $\alpha 3-\alpha 4$ loop and the C-terminal helix a9 exhibit a decrease in RMSF.

The interface between $\alpha 4-\alpha 5$ loop and $\alpha 7$ has been identified as an important site for communication between the $\mathrm{N}$-terminal 
Fig. 4 Eltrombopag stabilizes inactive BAX structure. a Overlay of structures of BAX-EO complex from 10 nsec intervals from 0 to 100 nsec molecular dynamics (MD) simulation. EO color spectrum corresponds to time as described, BAX ribbon structure is colored gray with residues of interest represented as sticks for clarity. b, c Distance relative to the time of EO carboxylate-R145 (carbonyl carbon- $\zeta$-carbon) (b) and EO pyrazolone carbonylR134 (carbonyl oxygen- $\zeta$-carbon) (c). Blue shades represent individual MD simulation distances, and black represents mean of $n=3$ simulations. d, e Histogram representation of $\alpha$-carbon distance frequency during MD simulation between R134 and negatively charged residues D48 (d) and E44 (e) on $\alpha 1-\alpha 2$ loop. Data represent a mean of $n=3$ for both BAX and BAX-EO. Data represent a mean of $n=3$ simulations for both BAX and BAX-EO. f Percentage change in root-mean square fluctuation (RMSF) of BAX-EO versus BAX is plotted with respect to BAX residue number. The color gradient is representative of change in RMSF, with blue and red corresponding to decrease and increase in RMSF, respectively. Data represent a mean of $n=$ 3 simulations for both BAX and BAX-EO. The average percentage change is represented by the dotted black line, with \pm SD represented as red and blue dashed lines. Residues associated with the $\mathrm{N}$-terminal trigger site, $\mathrm{BH}$ domain, canonical site, and transmembrane domain are highlighted in yellow, green, gray, and blue, respectively. $\mathbf{g}$ Changes in structure and dynamics of $\alpha 7 / \alpha 4-\alpha 5$ loop interface: representative $\alpha$-carbon distance frequency histogram for F105-Q155 (left), transparent surface with ribbon representation of $\alpha 7 / \alpha 4-\alpha 5$ loop interface with residues of note highlighted in blue (center), and graphical representation of distances between residues at $\alpha 7 / \alpha 4-\alpha 5$ loop interface (right). $\mathbf{h}$ Changes in structure and dynamics of the canonical site opening formed by $\alpha 3$, loop 3, $\alpha 4$, and $\alpha 9$ : representative $\alpha$-carbon distance frequency histogram for T85-K189 (left), transparent surface with ribbon representation of canonical site opening with residues of note highlighted in blue (center), and graphical representation of distances between residues at the canonical site opening (right). Distances represent the mean difference of $n=3$ BAX and BAX-EO MD simulations ( $\mathbf{g}$, $\mathbf{h}$ ), Distance plotted with respect to time and distance frequency histograms for all distances are available in supplementary figures as referenced. Source data for this figure is provided.

trigger site and the C-terminal canonical site ${ }^{18,40}$. Crystal structures of inactive BAX mutants (P168G and W139A) display changes in the interface of $\alpha 4-\alpha 5$ loop and $\alpha 7$, with notable changes in the conformation of F105 and W151. We measured the distances between $\alpha 4-\alpha 5$ loop and $\alpha 7$ and observed reduced distances between most of the residues in the presence of EO with the exception of the distance between F105 to W151, which increases (Fig. 4g and Supplementary Fig. 9). Furthermore, we observed changes in the distances between R89 and W139 on a4 and $a 6$, respectively, as well as in the distances between R89 and F93 on $a 6$, both of which showed changes in the inactive BAX crystal structures ${ }^{40}$ (Supplementary Fig. 10). The MD data strongly agree with the crystallographic structures of inactive BAX mutants and point to a potential mechanism by which binding at the trigger site propagates conformational changes throughout BAX.

The $a 3-\alpha 4$ loop and $\alpha 9$ form what can be considered as the opening of the canonical site. In order for BAX to translocate to the mitochondria, $\alpha 9$ must partially dissociate from the canonical site of $\mathrm{BAX}^{18,19,40}$. To evaluate this, we measured the distances between four residues forming the boundaries of the opening to the canonical site (Fig. $4 \mathrm{~h}$ and Supplementary Fig. 11). All of the distances measured were reduced. By approximating the canonical site opening as two triangles, we were able to calculate the approximate canonical site opening area of BAX as $113 \AA^{2}$ and the BAX-EO complex as $102 \AA^{2}$, a reduction of $\sim 9 \%$. Taken together, the MD data suggest that EO binding at the BAX trigger site induces direct and distal conformational changes consistent with stabilization of the inactive soluble BAX structure. These include changes in the interfaces of $\alpha 4$ and $\alpha 6$ as well as $a 4-\alpha 5$ loop and $\alpha 7$, which may allosterically couple the trigger site to $\alpha 9$ and the canonical site.

To independently assess the results of MD simulations, we measured paramagnetic relaxation enhancement (PRE) effects on ${ }^{15} \mathrm{~N}$-labeled BAX caused by a soluble paramagnetic probe, hyTEMPO, in the presence and absence of EO. The hy-TEMPO probe is a small sparsely functionalized molecule that can bind nonspecifically to solvent-exposed surfaces and pockets on the surface of BAX (Supplementary Fig. 12). We observed that the presence of EO altered the PRE effects not only by directly blocking hy-TEMPO binding to the trigger site but by altering the surface topology of BAX (Fig. 5a). Mapping these changes to the surface of BAX revealed that EO binding protected the trigger site residues in direct contact with EO based on the docking pose, particularly around the hydrophobic pocket formed between al and $a 6$ (Fig. 5b, c). As expected, PRE effects on $\alpha 1-\alpha 2$ loop were unchanged in the presence of EO, consistent with this loop remaining closely associated with the trigger site. Furthermore, reduction in PRE effects were observed on residues surrounding the interface of $\alpha 7$ and $\alpha 4-\alpha 5$ loop as well as internal residues of the canonical sites, such as $\alpha 3, \alpha 5$, and $\alpha 9$ (Fig. $5 \mathrm{~d}, \mathrm{e}$ ). This reduction in PRE effects at the interface of $\alpha 7$ and $\alpha 4-\alpha 5$ loop is consistent with the closer association predicted by MD simulations (Fig. 4). Furthermore, the reduction in PRE effects on internal canonical site residues is consistent with stabilization of $\alpha 9$ binding at the canonical site and a narrowing of the canonical site opening as suggested by MD simulations (Fig. 4). The PRE effects on these regions strongly corroborate our findings from biochemical, NMR, and molecular dynamics data and further support that binding events at the BAX trigger site can induce distal conformational changes in the canonical site via changes in $\alpha 4, a 4-\alpha 5$ loop, and $\alpha 7$, which result in inhibition of BAX activation and stabilization of the inactive BAX structure.

Eltrombopag inhibits BAX-mediated apoptosis. It is important to note that thrombopoietin (THPO)-receptor agonist activity of EO is highly specific to the human and chimpanzee THPOreceptors, making mouse cell lines ideal for studying EO modulation of BAX-dependent activity independent of THPOmediated effects ${ }^{42}$ (Supplementary Fig. 13a). First, we evaluated mitochondrial cytochrome $c$ release, a hallmark of BAX activation and BAX-dependent apoptosis. BIM-BH3-induced release of cytochrome $c$ was significantly inhibited by EO in BAK KO $\left(\mathrm{BAK}^{-1-}\right) \mathrm{MEF}$ providing direct evidence that $\mathrm{EO}$ can inhibit BAX-dependent cytochrome $c$ release (Fig. 6a). EO had no such effect in BAX $\mathrm{KO}\left(\mathrm{BAX}^{-/-}\right)$MEFs, strongly supporting BAX specificity (Supplementary Fig. 13b, c). BAX KO and BAK KO MEFs exhibited similar sensitivity to BIM-BH3-induced cytochrome $c$ release (Supplementary Fig. 13d). Next, we evaluated mitochondrial translocation of cytosolic BAX upon treatment with either BIM-BH3 or staurosporine (STS) in BAK KO MEFs, and we determined that EO is capable of inhibiting BAX translocation (Fig. 6b, c and Supplementary Fig. 13e), consistent with in vitro results (Fig. $2 \mathrm{~g}-\mathrm{i}$ and Supplementary Fig. $3 \mathrm{~d}-\mathrm{g}$ ). Accordingly, EO inhibited STS-induced apoptosis mediated by caspase-3/7 activity in MEFs expressing only BAX, but it had no effect is MEFs expressing only BAK (Supplementary Fig. 13f, g). To confirm whether these effects are linked to direct target engagement of BAX, we performed Cellular Thermal Shift Assay (CETSA) in BAK KO MEFs. CETSA showed that EO indeed binds BAX in cells by lowering its $\mathrm{T}_{M}$ by $9{ }^{\circ} \mathrm{C}$ (Fig. $6 \mathrm{~d}$ and Supplementary Fig. 14a). Interestingly, previous studies also have 


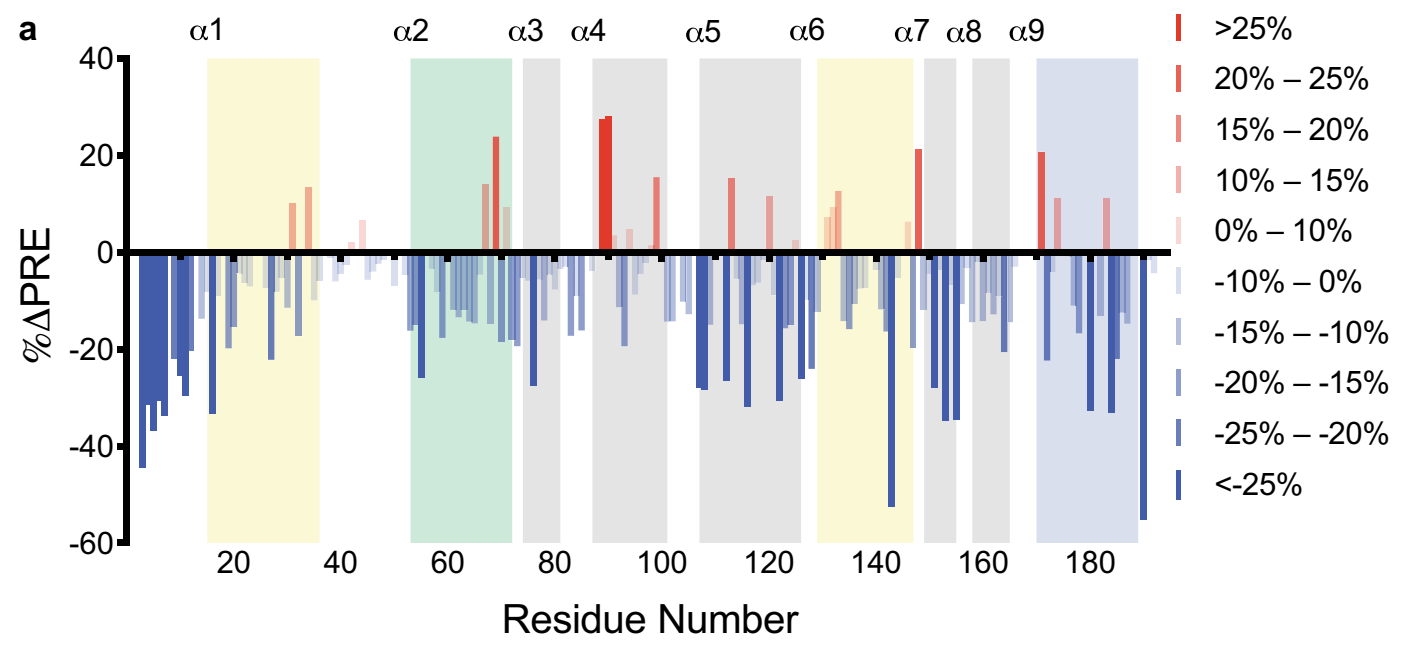

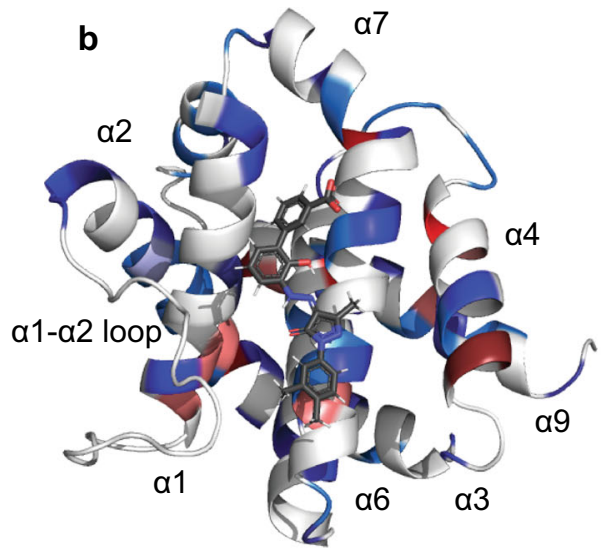

N-Terminal View

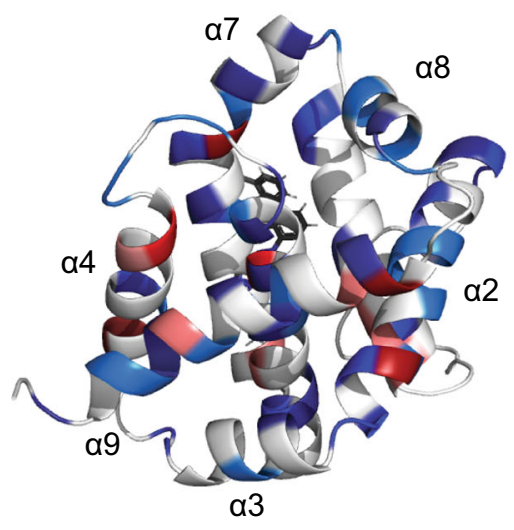

C-Terminal View

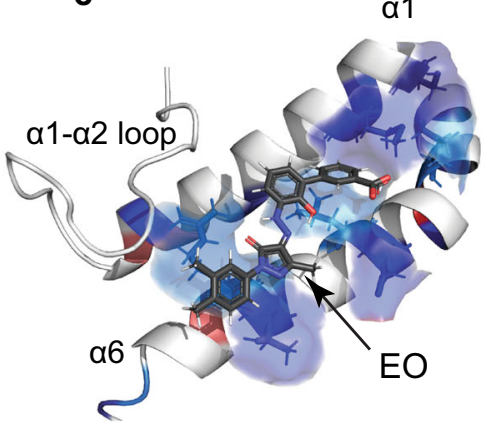

EO Binding Surface

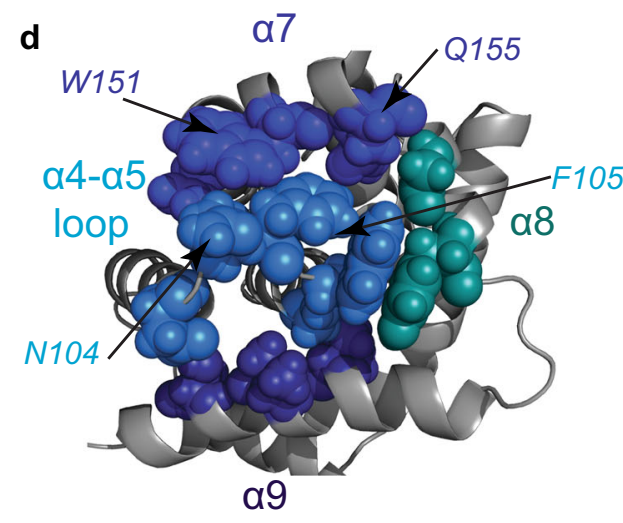

$\alpha 7-\alpha 4-\alpha 5$ loop Interface

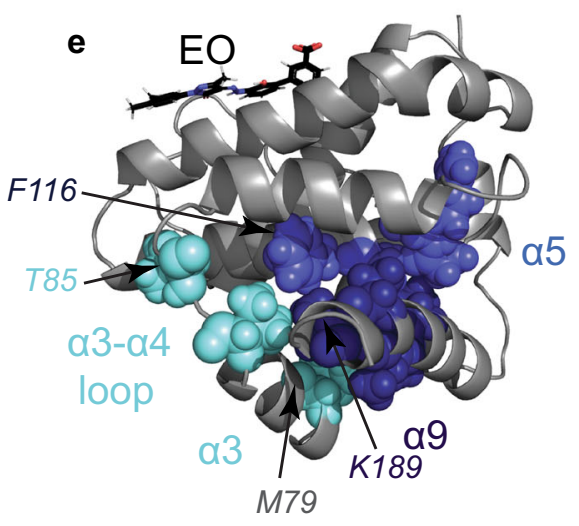

Canonical Site

Fig. 5 NMR-based evidence of Eltrombopag-mediated BAX inhibition. a EO-induced differences in peak intensity ratio (PRE) of peaks in the presence and absence of the soluble paramagnetic probe hydroxyl-TEMPO. Percentage change in PRE is plotted with respect to BAX residue number. Residues that exhibit increased or decreased PRE are labeled in shades of red and blue, respectively, the color gradient corresponding to the figure key. Residues associated with the $\mathrm{N}$-terminal trigger site, BH3 domain, canonical site, and transmembrane domain are highlighted in yellow, green, gray, and blue, respectively. b Ribbon representation of BAX-EO complex with mapping of residues undergoing significant differences in PRE as shown in (a). c Ribbon representation of EO-binding site with residues undergoing significant change in PRE colored corresponding to (a) and represented as a transparent surface with stick representation of residues. $\mathbf{d}$, e Cluster of residues about the $\alpha 7 / \alpha 4-\alpha 5$ loop interface (d) and canonical site (e) which exhibited reduced PRE in the presence of EO. Colors correspond to the location of the residue within the tertiary structure of BAX as indicated by the figure labels. Source data for this figure is provided. 


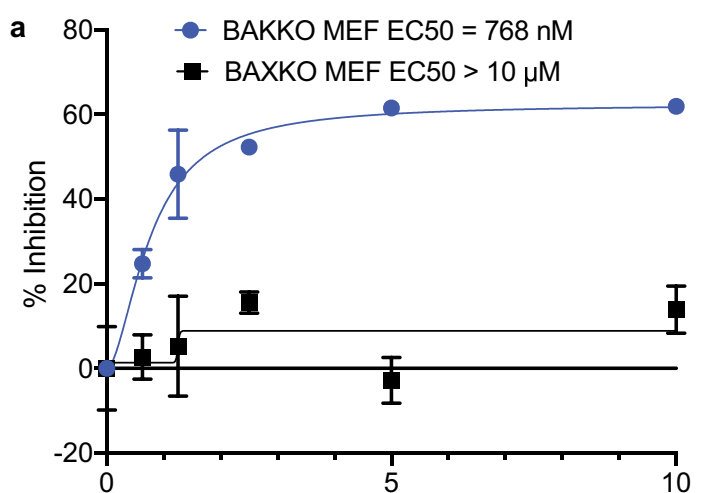

[EO] $\mu \mathrm{M}$

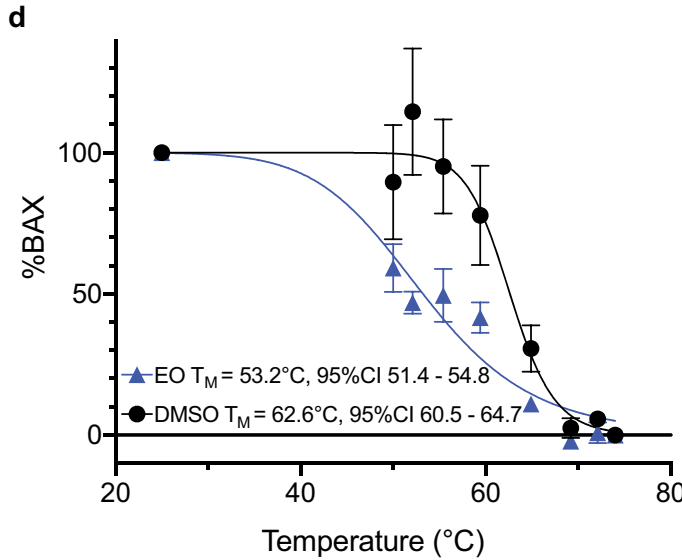

e

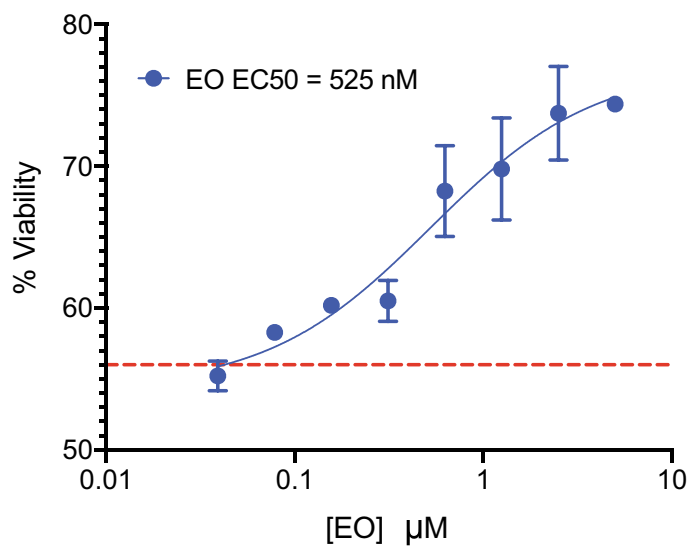

demonstrated that inactive BAX mutants can display dramatically reduced $T_{M}$ despite their resistance to activation by $\mathrm{BH} 3$ activators, highlighting a critical distinction between the controlled conformational changes of BAX activation and protein unfolding ${ }^{41,43}$. Furthermore, recombinant BAX displayed a comparable reduction in $\mathrm{T}_{\mathrm{M}}$ in the presence of $\mathrm{EO}$, strongly supporting the CETSA observations (Supplementary Fig. 14b, c). Finally, we determined if EO is capable of rescuing cell death in cells expressing both BAX and BAK. We treated 3T3 cells, a murine fibroblast cell line, with a combination of clinical BH3mimetics ABT-263 (Navitoclax) and S63845, which only in
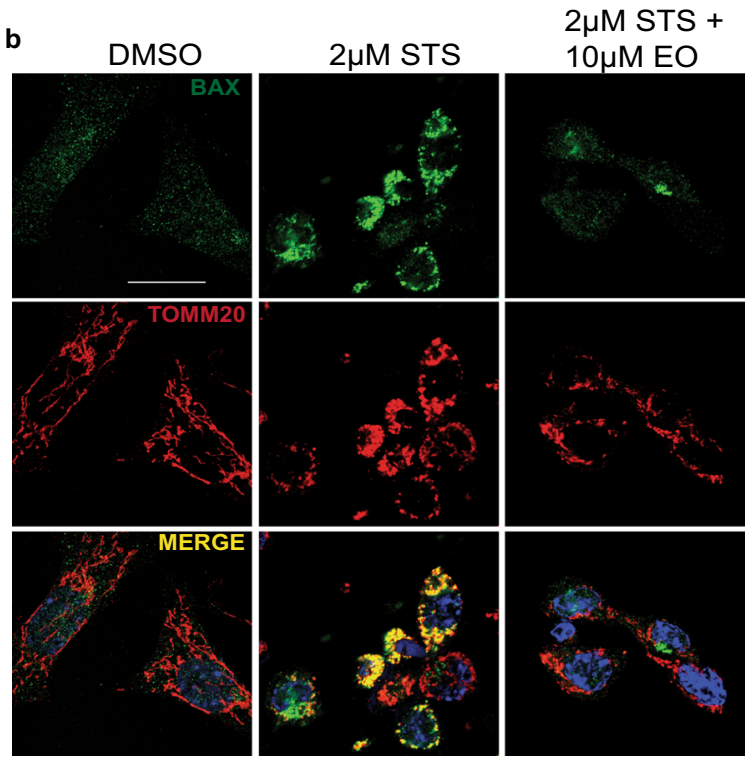

c
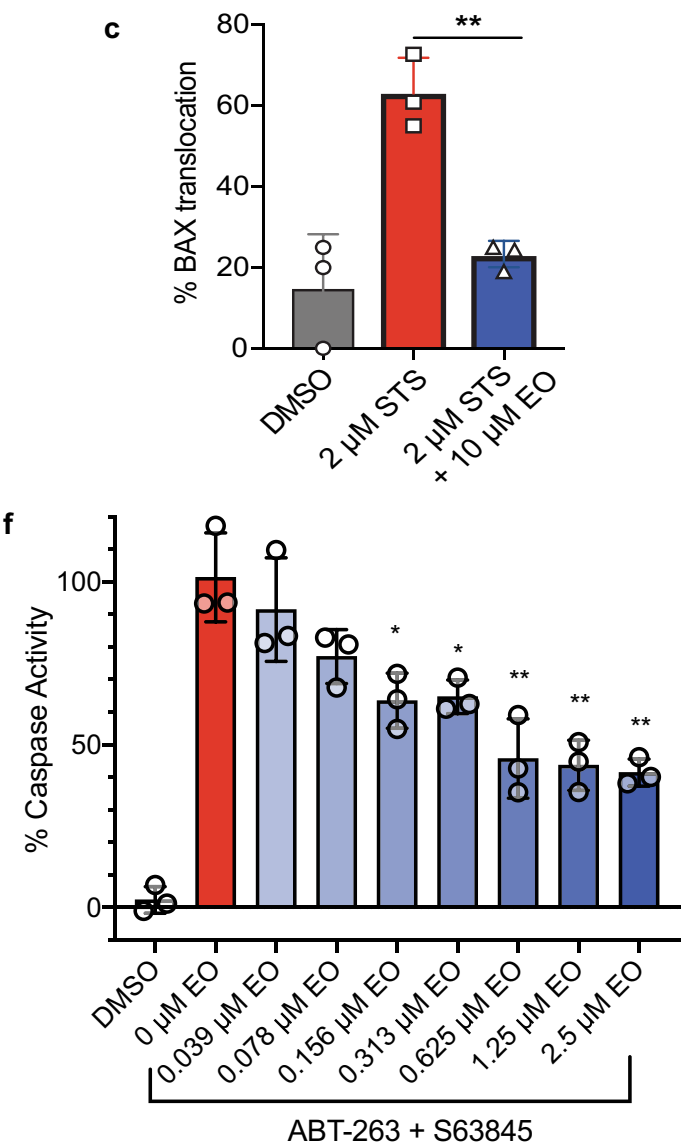

combination cause significant cytotoxicity in fibroblast cells (Supplementary Fig. 14d). Strikingly, cells treated with EO exhibited a dose-dependent rescue of cell viability as well as a corresponding significant reduction of apoptosis mediated by caspase-3/7 activity (Fig. 6e, f). Although EO does not inhibit BAK-mediated cytochrome $c$ release and apoptosis, it is capable of inhibiting cell death in cells expressing both BAK and BAX. It could be that in these cells, BAX is predominant in mediating apoptosis or EO inhibition of BAX frees more anti-apoptotic BCL-2 proteins to inhibit BAK in response to $\mathrm{BH} 3$ mimetics ${ }^{30,31}$. While we cannot definitely rule out other potential intracellular 
Fig. 6 Eltrombopag inhibits BAX-mediated cell death. a Dose-response EC50 curve for percentage inhibition of BIM-BH3-induced cytochrome $c$ release by EO in BAK KO (blue) and BAX KO (black) mouse embryonic fibroblasts with EC50 shown for clarity. Data represent mean of $n=3 \pm$ SEM and are representative of three independent experiments. $\mathbf{b}$ Confocal micrographs of BAK KO MEFs treated with DMSO, $2 \mu \mathrm{M} \mathrm{STS}$ for $4.5 \mathrm{~h}$ without or with $10 \mu \mathrm{M}$ EO $6.5 \mathrm{~h}$, respectively. BAX translocation is based on antibody-based detection of BAX and mitochondrial protein TOMM20. Representative confocal micrographs from three independent biological experiments. Scale bar, $20 \mu \mathrm{m}$. c Quantification of BAX translocation (\% of cells with BAX foci colocalizing with TOMM20 foci) in BAK KO MEFs induced by $2 \mu \mathrm{M}$ staurosporine (STS) and inhibited by $10 \mu \mathrm{M}$ EO. Data represent \pm SEM of three independent biological replicates. Two-sided $t$ test, ${ }^{\star \star \star \star} P<0.0001 ;{ }^{\star \star \star} P<0.001 ;{ }^{\star \star} P<0.01 ;{ }^{\star} P<0.05 ; n, P>0.05$. d Cellular thermal shift assay (CETSA) BAX melting curves for BAK KO MEFs treated with vehicle or $10 \mu \mathrm{M}$ EO. Data represent mean of $n=4 \pm$ SEM independent experiments. e Viability assay of $3 T 3$ cells upon treatment with $1 \mu \mathrm{M} \mathrm{ABT-263}$ and $1 \mu \mathrm{M}$ S63845 in the presence or absence of various doses of EO for $24 \mathrm{~h}$. Viability upon ABT-263 and S63845 combination in the absence of EO is indicated by the red dashed line. Data represent mean of $n=3 \pm S E M$ and are representative of three independent experiments. $\mathbf{f}$ Caspase-3/7 assay of 3T3 cells upon treatment with $1 \mu \mathrm{M}$ ABT-263 and $1 \mu \mathrm{M} S 63845$ in the presence or absence of various doses of EO for $4 \mathrm{~h}$. Data represent mean of $n=3 \pm$ SEM and are representative of three independent experiments. Two-sided $t$ test, ${ }^{\star \star \star \star \star} P<0.0001$;

${ }^{\star \star \star} P<0.001 ;{ }^{\star \star} P<0.01 ;{ }^{\star} P<0.05 ; \mathrm{ns}, P>0.05$. (left to right $P$ values were 0.0019 for $(\mathbf{c}), 0.0151,0.0122,0.0062,0.0031,0.0019$ for $(\mathbf{f})$ ). Source data for this figure is provided.

a

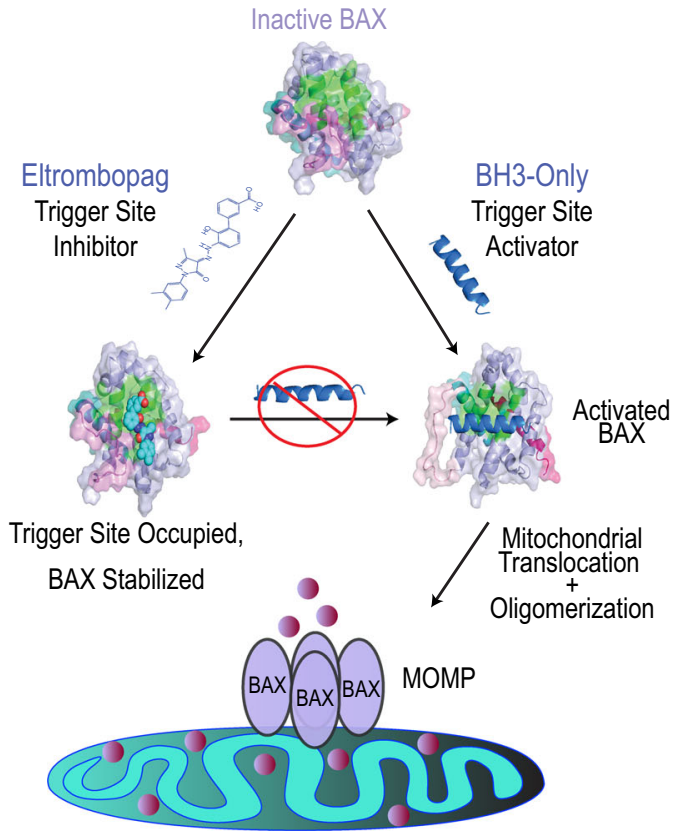

b
Trigger Site Activator Trigger Site Inhibitor
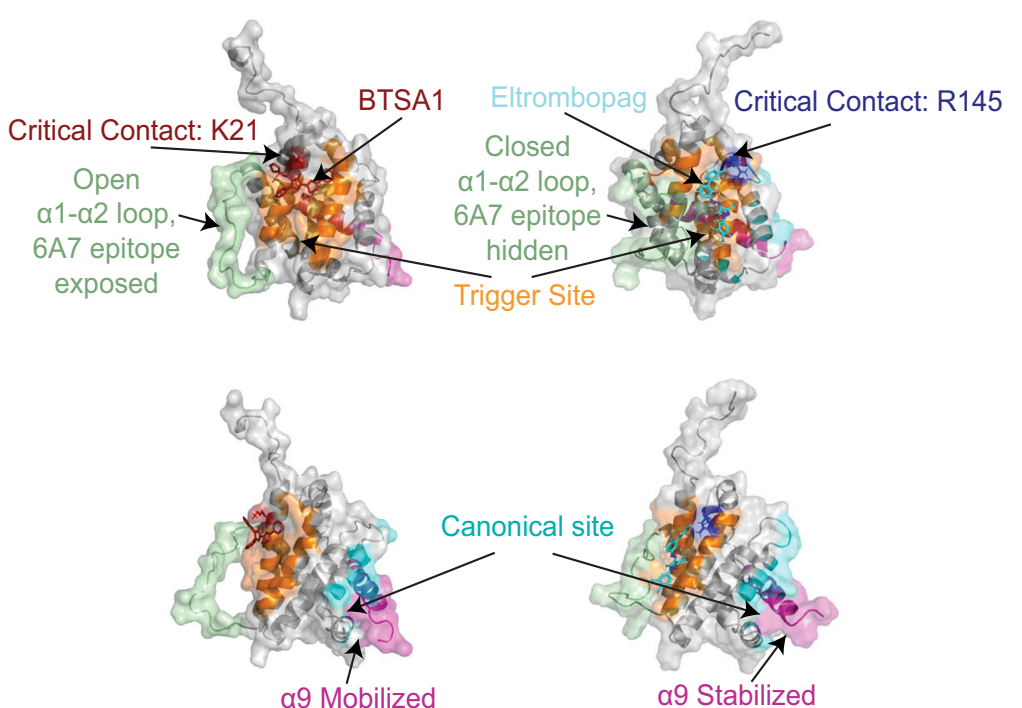

Fig. 7 Eltrombopag inhibits BAX activation by a unique twofold mechanism. a Eltrombopag's binding to the BAX trigger site competes off BH3-only activator proteins of $B A X$ and stabilizes an inactive structure of $B A X$ resulting in inhibition of $B A X$ activation, mitochondrial translocation, and BAXmediated MOMP. b BAX surface representations demonstrating distinct binding modes and associated conformational effects by the BAX trigger site inhibitor, Eltrombopag, and the BAX trigger site activator, BTSA1.

targets, our data strongly suggest EO targeting BAX in cells to inhibit cell death.

\section{Discussion}

Understanding the mechanism of BAX activation enables a deeper understanding of the critical function of BAX in apoptosis signaling and related pathways (e.g., mitochondrial-driven necrosis, mitochondrial dynamics) as well illuminates previously unknown targets and means to modulate BAX activity with drugs $28,29,31,34$. Here, our studies have identified EO, as a potent binder to the $\mathrm{BAX}$ trigger site and an effective BAX inhibitor (Fig. 7a). EO inhibits BAX activation by a unique twofold mechanism. BAX inhibition by $\mathrm{EO}$ is dependent on the concentration of $\mathrm{EO}, \mathrm{BAX}$, and $\mathrm{BH} 3$ activators, and $\mathrm{EO}$ directly engages the BAX trigger site, consistent with a direct competitive mechanism. Unlike trigger site activator small molecules and peptides that bind and displace loop $\alpha 1-\alpha 2$ from the trigger site as the critical first step in BAX activation, EO binds the BAX trigger site in a unique conformation that maintains the a1- $\alpha 2$ loop in an inactive closed conformation ${ }^{18,30,33,34}$. Likewise, BAX mutations at the trigger site that do not affect loop $\alpha 1-\alpha 2$ conformation can also inhibit BAX activation ${ }^{40}$. Furthermore, EO inhibits BAX by inducing discrete conformational changes in previously reported regions of allosteric communication such as $\alpha 7 / \alpha 4-\alpha 5$ loop which stabilizes $\alpha 9$ in the canonical site ${ }^{40-43}$. Thus, our data suggest a unique mechanism of BAX inhibition by EO that directly competes $\mathrm{BH} 3$-only proteins binding to BAX maintaining $\alpha 1-\alpha 2$ loop in an inactive closed conformation and simultaneously promoting allosteric conformational changes that stabilize the inactive soluble BAX structure.

The N-terminal BAX trigger site has been established as an important binding site for $\mathrm{BH} 3$-only proteins and small-molecule BAX activators that induce conformational changes throughout the BAX structure necessary for mitochondrial translocation, dimerization/oligomerization, and mitochondrial outer membrane permeabilization 10,17,19,33,34,40,41,43,44. This study provides further evidence that $\mathrm{BH}$-only proteins such as BID and BIM 
use the trigger site surface to induce BAX conformational activation. We identified EO by substructure similarity search of known small-molecule BAX activators. Despite some similar structural features to BAX activators, $\mathrm{EO}$ engages the trigger site with a unique binding mode distinct from BAX activators, using hydrophobic interactions with a shallow hydrophobic groove formed by residues of $\alpha 6, \alpha 1$, and the closed $\alpha 1-\alpha 2$ loop, and hydrogen bonds with R145 and R134 of a6. Here, we show that the BAX trigger site can also serve as a site of inhibition by small molecules and it can interfere with the early stages of BAX activation, before the disengagement of the $\alpha 9$ from the canonical groove and BAX mitochondrial translocation (Fig. 7b). Accordingly, our study offers a blueprint for rational drug design of a distinct class of BAX inhibitors using EO's mechanism of action of targeting BAX.

Our data contribute to the growing body of work highlighting bifunctional allosteric communication between the BAX trigger site and canonical site. Allostery has been proposed with activator $\mathrm{BH} 3$ peptides and small molecules such as BAM7 and BTSA1 that engage the BAX trigger site and promote conformational changes leading to the release of $\alpha 9$ from the opposite surface of $\mathrm{BAX}^{18,33,34}$. Allosteric BAX inhibitors (BAIs) and fragments that sensitize BAX to activation bind to adjacent sites while exhibiting opposite functional effects ${ }^{30,45}$. Furthermore, the BAX sensitizing fragments and the cytomegalovirus vMIA peptide compete for an identical binding site, yet the vMIA peptide inhibits BAX activity ${ }^{38,45}$. Synthetic antibodies recognizing the $\mathrm{N}$-terminal BAX trigger site in a way that prevents opening of loop $\alpha 1-\alpha 2$ have also served to inhibit BAX activation by blocking activator binding ${ }^{44}$. Furthermore, the BCL-2 BH4 domain inhibits BAX by binding to a unique site on the surface directly between the Nterminal trigger site and vMIA site ${ }^{39}$. Lastly, antibody $3 \mathrm{C} 10$ has an inhibitory effect to BAX by engaging the $\alpha 1-\alpha 2$ loop and favoring the allosteric sequestration of $\alpha 9$ in the canonical groove ${ }^{40,46}$. Therefore, it is possible that most if not all of the BAX surface could serve as a site of activation or inhibition given the appropriate interactions with a small molecule, peptide, or antibody/protein. Fine-tuning of the pro-apoptotic function of BAX via multiple structural mechanisms is critical in a physiologic context but could potentially be exploited pharmacologically to induce or inhibit cell death.

To our knowledge, EO is the first FDA-approved molecule with the ability to modulate BAX activity directly. The fact that $\mathrm{EO}$ is a well-tolerated orally bioavailable molecule inspires confidence in the potential of BAX inhibition as a therapeutic strategy for diseases of aberrant cell death. Interestingly, platelets isolated from patients treated with $\mathrm{EO}$ exhibited increased resistance to ABT-263-induced cell death ${ }^{47}$. This was not observed in patients treated with the fusion protein thrombopoietin receptor agonist romiplostin ${ }^{47}$, suggesting it is possible that $\mathrm{EO}$ inhibition of $\mathrm{BAX}$ contributes to its therapeutic activity. Although increased platelet levels after several days of continuous treatment with EO could prevent the repurposing of $\mathrm{EO}$ as a $\mathrm{BAX}$ inhibitor, evaluation of EO's ability to inhibit clinically relevant BAX-mediated cell death induced by acute apoptotic stimuli should be the subject of a future investigation. Furthermore, EO may serve as a powerful chemical tool for investigating the role of BAX in a variety of homeostatic and pathologic conditions and for drug design tailored to BAX inhibition.

\section{Methods}

Reagents. Hydrocarbon-stapled peptides corresponding to the $\mathrm{BH} 3$ domain of BIM, BIM SAHB: FITC-Ahx-EIWIAQELRS5IGDS5FNAYYA-CONH, where S5 represents the non-natural amino acid inserted for olefin metathesis, were synthesized and purified at $>95 \%$ purity by CPC Scientific Inc. Peptides corresponding to the $\mathrm{BH} 3$ domain of BIM, BIM-BH3, Ac-RPEIWIAQELRRIGDEFNAYYARR, was synthesized by GenScript at $>95 \%$ purity. Recombinant tBID in $>95 \%$ purity by SDS-PAGE under reducing conditions was purchased by R\&D Systems (cat. \# 882-B8-050). Eltrombopag (cat. \# 100941) and Eltrombopag methyl ester (cat. \# SC498745) were purchased from Medkoo Biosciences and Santa Cruz Biotechnology respectively and their molecular identity and purity $>98 \%$ was confirmed by NMR. ABT-263 (cat. \# S1001) in >99\% purity, and S63845 (cat. \# A8737) in $98 \%$ purity were purchased from Selleckchem, and APExBIO, respectively. Compounds were stored as powdered, reconstituted into 100\% DMSO, and diluted as described.

Production of recombinant BAX. Human full-length (1-192) wild-type BAX was cloned in pTYB1 vector (New England BioLabs) between the NdeI and SapI restriction sites. Mutations were generated using the QuickChange Lightning sitedirected mutagenesis kit (Agilent). Recombinant proteins were expressed in BL21 (DE3) CodonPlus (DE3)-RIPL, grown in Luria Broth media and induced with 1 $\mathrm{mM}$ isopropyl $\beta$-d-1-thiogalactopyranoside. The bacterial pellet was resuspended in lysis buffer $(20 \mathrm{mM}$ Tris- $\mathrm{HCl}$ pH 7.6, $250 \mathrm{mM} \mathrm{NaCl}, 1 \mathrm{mM}$ EDTA, and Roche complete EDTA-free protease inhibitor cocktail), lysed by high-pressure homogenization, and clarified by ultracentrifugation at $45,000 \times \mathrm{g}$ for $45 \mathrm{~min}$. The supernatant was applied to $5 \mathrm{ml}$ of pre-equilibrated chitin beads (New England BioLabs) in a gravity-flow column, and washed with three column volumes of lysis buffer. BAX was cleaved by overnight incubation using $50 \mathrm{mM}$ DTT in lysis buffer. Cleaved BAX was eluted with lysis buffer, concentrated with a Centricon spin concentrator (Millipore), and purified by gel filtration using a Superdex 75 10/300 GL, column (GE Healthcare Life Sciences), pre-equilibrated with gel filtration buffer $(20 \mathrm{mM}$ HEPES, $150 \mathrm{mM} \mathrm{KCl}, \mathrm{pH} 7.2)$ at $4^{\circ} \mathrm{C}$. Fractions containing BAX monomer are pooled and concentrated using a $10-\mathrm{KDa}$ cutoff Centricon spin concentrator (Millipore) for prompt use in biochemical and structural studies.

Fluorescence polarization binding assays. Fluorescence polarization assays (FPA) were performed as previously described ${ }^{34}$. Direct binding isotherms of BIM-SAHB were measured by incubated FITC-BIM-SAHB $(25 \mathrm{nM})$ with serial dilutions of full-length BAX alone or in the presence of 0.5 or $1 \mu \mathrm{M} \mathrm{EO}$. Competition binding assays were performed by titrating EO into BAX $(150 \mathrm{nM})$ and FITC-BIM-SAHB (25 nM). Measurements were taken at 10-min intervals over 60 min on a TECAN F200 PRO microplate reader. Reported curves represent a 10 min time point. $\mathrm{K}_{\mathrm{D}}$ values and $\mathrm{IC}_{50}$ were determined using GraphPad Prism nonlinear fit four-parameter agonist or antagonist versus response with restraints for $100 \%$ and $0 \%$ bound calculated by the $\mathrm{mP}$ of saturated BAX + FITCBIM-SAHB and FITC-BIM-SAHB alone.

Microscale thermophoresis. Recombinant BAX C62S C126S S5C (4C), previously established for evaluating $\mathrm{BAX}^{30}$ binding compounds with MST, or BAX C62S C126S S5C R134E R145E (R134E R145E 4C) was labeled at cysteine using the Monolith Protein Labeling Kit Red Maleimide (NanoTemper Technologies) according to the instructions of the manufacturer ${ }^{31}$. Briefly, $10 \mu \mathrm{M}$ protein was incubated with 0.9 equivalents of dye in MST buffer $(100 \mathrm{mM}$ potassium phosphate, $\mathrm{pH} 7.4,150 \mathrm{mM} \mathrm{NaCl})$ in the dark at room temperature $\left(22-25^{\circ} \mathrm{C}\right)$ for $1 \mathrm{~h}$ Unreacted dye was quenched using $5 \mathrm{mM}$ DTT and removed using the manufacturer provided buffer exchange column. To determine the $\mathrm{K}_{\mathrm{D}}$ of $\mathrm{BAX}$ to $\mathrm{EO}, 50$ $\mathrm{nM}$ labeled BAX was incubated with increasing concentrations of EO in MST buffer supplemented with $0.25 \%$ CHAPS. Samples were loaded into standard glass capillaries (Monolith NT.155 Capillaries) and analyzed by MST using a Monolith NT.115 Blue/Red, LED power, and IR laser power of $80 \%$. The fraction bound and error was generated by NanoTemper software (MO.Affinity Analysis 3 ) and $K_{D}$ values were determined using GraphPad Prism nonlinear fit four-parameter agonist versus response with restraints for 0 and 1 fraction bound.

Liposomal permeabilization assay. Lipids (Avanti Polar Lipids) at the following ratio, phosphatidylcholine $48 \%$, phosphatidylinositol $10 \%$, dioleoyl phosphatidylserine $10 \%$, phosphatidylethanolamine, $28 \%$, and tetraoleoyl cardiolipin $4 \%$, were mixed in a total of $1 \mathrm{mg}$, dried and resuspended in $10 \mathrm{mM}$ HEPES, pH 7, 200 $\mathrm{mM} \mathrm{KCl}$, and $5 \mathrm{mM} \mathrm{MgCl} 2$ with $12.5 \mathrm{mM}$ 8-aminonaphthalene-1,3,6-trisulfonic acid (ANTS) dye and $45 \mathrm{mM}$ p-xylene-bis-pyridinium bromide (DPX) quencher (Molecular Probes) using a water bath sonicator. Liposomes were formed by extrusion of the suspension using Avanti Mini-Extruder (cat \# 610000) with polycarbonate membranes of $0.1-\mu \mathrm{m}$ pore size (Avanti Polar Lipids). ANTS/DPX encapsulated liposomes were purified from non-encapsulated ANTS/DPX by gel filtration of a $10 \mathrm{~mL}$ CL2B-Sepharose (GE Healthcare Life Sciences) gravity-flow column. BAX (50-250 nM) was combined with tBID, BIM-BH3, and EO at the indicated concentrations to a volume of $90 \mu \mathrm{L}$. Reactions were initiated by the addition of $10 \mu \mathrm{L}$ of the encapsulated ANTS/DPX liposome stock. ANTS/DPX release was quantified based on the increase in fluorescence intensity that occurs when the ANTS fluorophore is separated from the DPX quencher upon release from the liposomes into solution. Fluorescence $(\lambda \mathrm{ex}=355 \mathrm{~nm}$ and $\lambda \mathrm{em}=520 \mathrm{~nm})$ was measured at $1 \mathrm{~min}$ of intervals at room temperature $\left(22-25^{\circ} \mathrm{C}\right)$ indicated using a Tecan Infinite M1000 plate reader. In the case of heat activation, reactions were set up as described in the absence of $\mathrm{tBID}$ or BIM-BH3, and experiments were recorded at $42{ }^{\circ} \mathrm{C}$. The percentage release of ANTS/DPX at any given time point was 
calculated as percentage release $=\left(\left(\mathrm{F}-\mathrm{F}_{0}\right) /\left(\mathrm{F}_{100}-\mathrm{F}_{0}\right)\right)(100)$, where $\mathrm{F}_{0}$ and $\mathrm{F}_{100}$ are baseline and maximal fluorescence, respectively. Triton X-100 (1\%) was used to determine the maximum amount of liposomal release per assay and is set to $100 \%$.

Liposomal translocation assay. Lipids (Avanti Polar Lipids) at the following ratio, phosphatidylcholine $48 \%$, phosphatidylinositol $10 \%$, dioleoyl phosphatidylserine $10 \%$, phosphatidylethanolamine, $28 \%$, and tetraoleoyl cardiolipin $4 \%$, were mixed in a total of $1 \mathrm{mg}$, dried and resuspended in $10 \mathrm{mM}$ HEPES, pH 7, $200 \mathrm{mM} \mathrm{KCl}$, and $5 \mathrm{mM} \mathrm{MgCl}$. The resulting slurry was vortexed for $10 \mathrm{~min}$ and sonicated in a sonicating water bath for $10 \mathrm{~min}$. Liposomes were formed by extrusion of the suspension using Avanti Mini-Extruder with polycarbonate membranes of $0.1-\mu \mathrm{m}$ pore size (Avanti Polar Lipids) followed by passage through a CL2B-Sepharose column (GE Healthcare). Recombinant wild-type BAX was labeled at cysteine by overnight incubation at $4{ }^{\circ} \mathrm{C}$ with ten equivalents of iodoamino-NBD (IANBD, ThermoFisher) and three equivalents of TCEP to maintain reduced cysteine. Labeled BAX (BAX-NBD) was separated from unreacted IANBD by gel filtration (Econo-Pac 10 DG desalting column, BioRad) and used immediately. Translocation reactions were performed by combining $800 \mathrm{nM}$ BAX with $1 \mu \mathrm{M}$ BIM-BH3 or $200 \mathrm{nM}$ tBID in the presence and absence of varying doses of EO. Reactions were initiated by the addition of $10 \mu \mathrm{L}$ of the liposome stock. The NBD fluorophore exhibits low fluorescence in solution due to quenching by water. Upon BAX-NBD translocation, the NBD fluorophore is excluded from bulk water through contact with the liposomal membrane leading to an increase in fluorescence intensity. Fluorescence $(\lambda \mathrm{ex}=475 \mathrm{~nm}$ and $\lambda \mathrm{em}=530 \mathrm{~nm})$ was measured at 1 -min intervals at $37^{\circ} \mathrm{C}$ indicated using a Tecan Infinite M1000 plate reader. The percentage translocation at any given time point was calculated as percentage translocation $=$ $\left[\left(\left(\mathrm{F}-\mathrm{F}_{0}\right) /\left(\mathrm{F}_{100}-\mathrm{F}_{0}\right)\right)(100)\right]-\left[\left(\left(\mathrm{F}_{\mathrm{S}}-\mathrm{F}_{\mathrm{S} 0}\right) /\left(\mathrm{F}_{\mathrm{S} 100}-\mathrm{F}_{\mathrm{S} 0}\right)\right)(100)\right]$, where $\mathrm{F}_{0}$ and $\mathrm{F}_{100}$ are baseline and maximal fluorescence, respectively, and $\mathrm{F}_{\mathrm{S}}, \mathrm{F}_{\mathrm{S} 0}$, and $\mathrm{F}_{\mathrm{S} 100}$ are the current fluorescence, baseline fluorescence, maximal fluorescence of solution BAX incubated in the absence of liposomes. The subtraction of the percent translocation of solution BAX is required to correct for NBD-fluorescence bleaching that occurs throughout the reaction. Triton X-100 $(0.1 \%)$ was used to determine the maximum amount of liposomal translocation per assay and is set to $\mathrm{F}_{100} 100 \%$.

BAX conformation change assay using anti-6A7 immunoprecipitation. Exposure of the 6A7 epitope of BAX was assessed by immunoprecipitation with a 6A7domain-specific antibody purchased from Santa Cruz (SC-23959). Protein G beads $(50 \mu \mathrm{L}$, Santa Cruz) were washed three times with $3 \%$ BSA in PBS and incubated with $15 \mu \mathrm{L} 6 \mathrm{~A} 7$ antibody at $4^{\circ} \mathrm{C}$ for $1 \mathrm{hr}$. Recombinant full-length BAX $(10 \mu \mathrm{M})$ was incubated with four equivalents of BIM-BH3 peptide alone and in the presence of five or ten equivalents of $\mathrm{EO}$ for $15 \mathrm{~min}$ at room temperature. Incubation of fulllength recombinant $\mathrm{BAX}$ with $0.1 \%$ Triton $\mathrm{X}$ served as a positive control for exposure of the 6A7 epitope. After incubation, $10 \mu \mathrm{L}$ of each reaction was transferred to the protein $\mathrm{G}$ beads pre-loaded with anti-6A7 antibody and $1 \mu \mathrm{L}$ was reserved as a loading control. After 90 min of incubation at $4{ }^{\circ} \mathrm{C}$, beads were collected and washed three times with $500 \mu \mathrm{L}$ of $3 \%$ BSA in PBS and solubilized with $25 \mu \mathrm{L}$ LDS/DTT loading buffer. Samples were resolved by SDS-PAGE electrophoresis and western blot analysis with a BAX monoclonal antibody (Cell Signaling, Cat. \# 2772) 1:1000.

\section{Western blotting and protein quantification. BAX samples were electro-} phoretically separated on $4-12 \%$ NuPage (Invitrogen) gels, transferred to mobilonFL PVDF membranes (Millipore), and subjected to immunoblotting.(For visualization of proteins with Odyssey Infrared Imaging System (LI-COR Biosciences), membranes were blocked in PBS containing $2.5 \%$ milk powder. Primary BAX monoclonal antibody (Cell Signaling, Cat. \#2772) was incubated overnight at $4{ }^{\circ} \mathrm{C}$ in a 1:1000 dilution. After washing, membranes were incubated with an IRdye800conjugated goat anti-mouse IgG secondary antibody (LI-COR Biosciences, cat. \# 925-32210) in a 1:5000 dilution. Protein was detected with Odyssey Infrared Imaging System. Densitometry of protein bands was acquired using an LI-COR Odyssey scanner. Quantification and analysis were performed using the western analysis tool from the Image Studio 3.1 software.

NMR samples and spectroscopy. The uniformly ${ }^{15} \mathrm{~N}$-labeled protein samples were prepared by growing the bacteria in a minimal medium, as previously described ${ }^{44}$. Unlabeled and ${ }^{15} \mathrm{~N}$-labeled protein samples were prepared in $50 \mathrm{mM}$ potassium phosphate, $50 \mathrm{mM} \mathrm{NaCl}$ solution at $\mathrm{pH} 6.0$ in $10 \% \mathrm{D}_{2} \mathrm{O}$. All experiments were performed using an independent sample for each experimental measurement as a $400 \mu \mathrm{L}$ sample in a $5-\mathrm{mm}$ Shigemi; all samples were DMSO matched with $2 \% \mathrm{~d}_{6}$-DMSO. Correlation ${ }^{1} \mathrm{H}-{ }^{15} \mathrm{~N}-\mathrm{HSQC}$ spectra were recorded on ${ }^{15} \mathrm{~N}$ labeled BAX at $50 \mu \mathrm{M}$ in the presence and absence of $100 \mu \mathrm{M}$ of EO. NMR spectra were acquired at $25^{\circ} \mathrm{C}$ on a Bruker $600 \mathrm{MHz}$ spectrometer equipped with a cryoprobe, processed using TopSpin and analyzed using NMRView. BAX crosspeak assignments were applied as previously reported ${ }^{17}$. The weighted average chemical shift perturbation (CSP) was calculated as $\left.\sqrt{ }\left(\Delta \delta^{1} H\right)^{2}+\left(\Delta \delta^{15} \mathrm{~N} / 5\right)^{2}\right) / 2$ in p.p.m. The absence of a bar indicates no chemical shift difference, the presence of proline, or a residue that is overlapped or missing and therefore not used in the analysis. The significance threshold for backbone amide chemical shift changes was calculated based on the average chemical shift across all residues plus 0.5 or $1 \mathrm{s.d} \mathrm{d}^{48}$.
The solvent-accessible surface area was probed by the addition of $10 \mathrm{mM}$ hyTEMPO (Sigma) to $50 \mu \mathrm{M}{ }^{15} \mathrm{~N}$-labeled BAX with and without $100 \mu \mathrm{M}$ EO measured using standard ${ }^{1} \mathrm{H}-{ }^{15} \mathrm{~N}-\mathrm{HSQC}$ with an increased recycle delay of $2 \mathrm{~s}^{30,49} \mathrm{PRE}$ was calculated as the ratio of peak intensities of BAX in the presence of hy-TEMPO to BAX without hy-TEMPO (\% intensity). Mapping of chemical shifts and PRE data onto the BAX structure was performed with PyMOL (Schrodinger, LLC, 2018-2019). The software was made available through the SBGrid collaborative network ${ }^{50}$.

NMR-based docking calculations and molecular dynamics. NMR-guided docking of EO into the NMR structure of BAX (PDB: 1F16: https://doi.org/ $10.2210 / \mathrm{pdb} 1 \mathrm{~F} 16 / \mathrm{pdb}$ ) was performed using induced-fit docking (IFD, Schrodinger, LLC, 2018) with extra precision (XP) and a binding site at the mid-point of residues K21, R134, and R145. EO was converted to 3D all atom structure using LIGPREP (Schrodinger, LLC, 2018) and assigned partial charges with EPIK (Schrodinger, LLC, 2018). Poses generated were consistent with NMR data and indicated a strong favoring of ionic interaction between the carboxylate of $\mathrm{EO}$ and a basic residue of BAX. Mutagenesis was used to elucidate the pose of EO on the trigger site of BAX. The pose consistent with mutant BAX liposomal release data was most consistent with NMR CSP data. This pose was subjected to three independent 100 nsec molecular dynamics (MD) simulations using DESMOND (DESMOND, version 3, Schrodinger, LLC, 2017). Three independent $100 \mathrm{~ns}$ MD simulations were also performed with the lowest energy BAX structure from the NMR ensemble (PDB 1F16: https://doi.org/10.2210/pdb1F16/pdb). MD runs were performed in a truncated octahedron SPC water box using OPLS_2005 force field, $300 \mathrm{~K}$, and the constant pressure of 1.0325 bar. Analysis of the trajectory was performed with MAESTRO simulation event analysis tools (Schrodinger, LLC, 2018). PyMOL (Schrodinger, LLC, 2018-2019) was used for preparing the highlighted poses. The $\% \triangle \mathrm{RMSF}$ for each residue was calculated as $\% \triangle \mathrm{RMSF}=$ $\left(\left(\mathrm{RMSF}_{\mathrm{EO}}-\mathrm{RMSF}_{\mathrm{ApO}}\right) 100 / \mathrm{RMSF}_{\mathrm{ApO}}\right)$, where RMSF $\mathrm{FO}_{\mathrm{EO}}$ was the RMSF of an individual MD simulation of EO docked into BAX and $\mathrm{RMSF}_{\mathrm{Apo}}$ is the average RMSF of the apo BAX simulation. Distance frequency histograms were prepared using GraphPad Prism frequency distribution analysis. The docked binding poses of BAM7, BTSA1, and EO within the BAX trigger site were used to calculate the binding energy of each compound using the MM-GBSA module ${ }^{51}$ in Maestro (Schrödinger, LLC, NY, 2018-2019). Docked complexes were analyzed with the MM-GBSA module using VSGB solvation model and OPLS3 force field.

Structural analysis. Structural analysis was performed in PyMOL (Schrödinger, LLC: NY, 2018-2019) and Maestro tools SiteMap, GLIDE, EPIC, and LIGPREP (Schrödinger, LLC, NY, 2018-2019).

Cytochrome $c$ release assay. $B A X^{-1-}$ or $B A K^{-1-}$ mouse embryonic fibroblasts were maintained in DMEM (Life Technologies) supplemented with $10 \%$ FBS, $100 \mathrm{U} / \mathrm{mL}$ penicillin/streptomycin, $2 \mathrm{mM}$ l-glutamine, and $0.1 \mathrm{mM}$ MEM nonessential amino acids. MEFs $\left(5 \times 10^{4}\right.$ cells/well $)$ were seeded in a 96 -well clear ubottom plate for 18-24 h. BAX or BAK expression was confirmed with immunoblot (anti-BAX antibody 2772S, Cell Signaling, 1:1000 dilution and anti-BAK antibody 06-536, Millipore, 1:1000 dilution). Media was removed and replaced with media lacking FBS, and cells were treated with varying doses of EO for $2 \mathrm{~h}$ at $37^{\circ} \mathrm{C}$. After incubation, the media was removed and replaced with $100 \mu \mathrm{L}$ reaction buffer modified from MEB buffer $(150 \mathrm{mM}$ mannitol, $10 \mathrm{mM}$ HEPES$\mathrm{KOH}$ pH 7.5, $50 \mathrm{mM} \mathrm{KCl}, 0.02 \mathrm{mM}$ EGTA, $0.02 \mathrm{mM}$ EDTA, 0.1\% BSA, $5 \mathrm{mM}$ succinate, $20 \mu \mathrm{g} / \mathrm{mL}$ oligomycin, $10 \mathrm{mM}$ DTT, and $0.00125 \%$ digitonin) with and without $5 \mu \mathrm{M}$ BIM-BH3 peptide and incubated at $30^{\circ} \mathrm{C}$ for $45 \mathrm{~min}$. After incubation, an additional $100 \mu \mathrm{L}$ of reaction buffer was added and the plate was gently tapped to mix. Cytochrome $c$ release was determined by decanting $50 \mu \mathrm{L}$ of the supernatant and analyzing with the rat/mouse cytochrome $c$ quantikine ELISA kit (R\&D Systems, MCT0) according to the recommended protocol. Percentage inhibition was normalized to BIM-BH3 peptide alone $(0 \%)$ and untreated cells $(100 \%)$.

In cell BAX translocation assays. BAK KO Cells $\left(1 \times 10^{6}\right.$ cells/well $)$ were seeded in $10-\mathrm{cm}$ clear bottom plate overnight in the media described above. The media was removed and replaced with media lacking FBS. Cells were then pre-treated with 10 or $20 \mu \mathrm{M} \mathrm{EO}$ as a $10 \mathrm{X}$ stock in $\mathrm{dH}_{2} \mathrm{O}$ or $\mathrm{dH}_{2} \mathrm{O}$ vehicle for $1 \mathrm{~h}$. After incubation, the media was removed, cells were washed with PBS, harvested and resuspended in $100 \mu \mathrm{L}$ reaction buffer modified from MEB buffer (with and without $10 \mu \mathrm{M}$ and $20 \mu \mathrm{M}$ BIM-BH3 peptide and incubated at $30^{\circ} \mathrm{C}$ for $1 \mathrm{~h}$. Reactions were then moved to ice and supplemented with an additional $0.025 \%$ digitonin for $10 \mathrm{~min}$. The cytosolic fraction was isolated by centrifugation for $10 \mathrm{~min}$ at $1500 \times \mathrm{g}$. The mitochondrial pellet was solubilized using PBS $+1 \%$ Triton X 100 for $1 \mathrm{hr}$ followed by centrifugation at $21,000 \times g$. Protein concentrations were normalized and resolved by SDS-PAGE electrophoresis and western blot analysis using 4-12\% NuPage (Life Technologies) gels, and analyzed by immunoblotting with anti-BAX antibody (2772S, Cell Signaling, 1:1000 dilution). VDAC1 (Cell Signaling, Cat. \# 4661, 1:1000 dilution) and b-Actin (Sigma, Cat. A1978, 1:5000 dilution) are used for loading control of mitochondrial and supernatant fractions respectively. For detection of BAX translocation using 
confocal microscopy, BAK KO MEFs were treated with $10 \mu \mathrm{M}$ EO at $\sim 70 \%$ confluence in media lacking FBS for $2 \mathrm{~h}$ prior to the addition of $10 \%$ FBS then and 2 $\mu \mathrm{M}$ of STS or DMSO for $4.5 \mathrm{~h}$. After treatments, cells were washed with PBS and fixed with $4 \%$ PFA for $15 \mathrm{~min}$. Then cells were permeabilized with $0.1 \%$ Triton X100 in PBS for 10 min. Cells were blocked with 5\% BSA in PBS-T and incubated overnight with primary antibodies as indicated; BAX (Cell Signaling Technology; 2772S, 1:100 dilution) and TOMM20 (Sigma; ST1705, 1:100 dilution). After incubation with primary antibodies, cells were washed with PBS and incubated with the appropriate mouse or rabbit secondary antibodies (ThermoFisher Scientific; A11008, A11030, 1:500 dilution) in the blocking solution. After PBS washes, coverslips were dipped in water and mounted on glass slides using Vectashield containing DAPI (Vector laboratories). Images were taken with Leica SP5 inverted confocal microscope. Data were analyzed with ImageJ.

Cell viability and caspase-3/7 activation assays. $3 \mathrm{~T} 3$ cells were maintained in media identical to that of MEFs. 3T3 cells were seeded $\left(1 \times 10^{4}\right.$ cells/well $)$ in 96 well opaque plates for $18-24 \mathrm{~h}$. The media was removed and replaced with media lacking FBS and cells were treated with $\mathrm{EO}$ as a $10 \times$ stock in $\mathrm{H}_{2} \mathrm{O}$ at the indicated doses for $2 \mathrm{~h}$ before addition of $10 \%$ FBS. Cells were then treated with $1 \mu \mathrm{M}$ each of ABT-263 and S63845. Caspase-3/7 activation was measured at $4 \mathrm{~h}$ by addition of the Caspase-Glo 3/7 chemiluminescence reagent in accordance with the manufacturer's protocol (Promega). Luminescence was detected by an F200 PRO microplate reader (TECAN). Percentage caspase activation was normalized to ABT-263 + S63845 alone (100\%) and untreated cells (0\%). Viability assays were performed at $24 \mathrm{~h}$ by addition of CellTiter-Glo according to the manufacturer's protocol (Promega). Luminescence was detected by an F200 PRO microplate reader (TECAN). Percentage viability was normalized to untreated cells $(100 \%)$. For caspase-3/7 assay in BAK KO and BAX KO MEFs, cells $\left(2.5 \times 10^{3}\right.$ cells/well) were seeded in a 384-well white plate and treated with EO as a 10× stock in DMSO at the indicated doses in the absense of FBS for $2 \mathrm{~h}$ prior to staurosporine treatment and addition of $10 \%$ FBS. Caspase- $3 / 7$ activation was measured at $6 \mathrm{~h}$ by addition of the Caspase-Glo 3/7 chemiluminescence reagent in accordance with the manufacturer's protocol (Promega). Luminescence was detected by a F200 PRO microplate reader (TECAN). Caspase assays were performed in at least triplicate and the data normalized to cell death stimulus-treated wells. Dilutions of EO or staurosporine were performed using a TECAN D300e Digital Dispenser from DMSO stocks.

Cellular thermal shift assays (CETSA). BAK KO MEFs were seeded in a $10-\mathrm{cm}$ dish for $18-24 \mathrm{~h}$ or until $\sim 80 \%$ confluent. The media was removed and replaced with media lacking FBS and cells were treated with $10 \mu \mathrm{M}$ EO as a $10 \times$ stock in $\mathrm{H}_{2} \mathrm{O}$ or vehicle for $2 \mathrm{~h}$. The media was then removed and cells were harvested using a cell scraper and washed twice with PBS. Cells were then resuspended in PBS to $6 \times 10^{6}$ cells $/ \mathrm{mL}$ and $50 \mu \mathrm{L}$ was transferred to PCR tubes. Cells were then heated in a Biorad C1000 Touch Thermal Cycler for 3 min using a temperature gradient $\left(50,52.1,55.4,59.4,64.9,69.2,72.1\right.$, and $\left.74^{\circ} \mathrm{C}\right)$. Cells remaining at room temperature $\left(25^{\circ} \mathrm{C}\right)$ served as a control. All cells were lysed by three cycles of freeze-thawing using liquid nitrogen. Samples were then centrifuged at $2 \times$ $10^{4} \times g$ for $15 \mathrm{~min}$. The supernatants were collected and resolved by SDS-PAGE with an N-terminal BAX antibody (Cell Signaling, 2772S). Samples were analyzed and quantified using a Li-Cor Odyssey Clx and normalized to $25^{\circ} \mathrm{C}(100 \%)$ and $74^{\circ} \mathrm{C}(0 \%)$

Calculation of recombinant BAX $\mathbf{T}_{\mathbf{M}}$. Purified recombinant BAX $(25 \mu \mathrm{M})$ was combined with DMSO or EO (1:10 BAX:EO) and loaded into Tycho NT.6 (NanoTemper Technologies) capillaries. First derivative $(330 / 350 \mathrm{~nm})$ melting point curves were generated automatically using the Tycho NT.6 (NanoTemper Technologies). Data were exported and to GraphPad PRISM software for analysis and visualization.

Statistical analysis. Statistical significance for pair-wise comparison of groups was determined by two-tailed Student's $t$ test using GraphPad PRISM software (GraphPad Inc., CA). $P$ values of less than 0.05 were considered significant.

Reporting summary. Further information on research design is available in the Nature Research Reporting Summary linked to this article.

\section{Data availability}

Data generated or analyzed during this study are included in this published article and its Supplementary Information files and are available from the corresponding author on a reasonable request. The following publicly available datasets were used in the production of this paper: PDB 1F16 [https://doi.org/10.2210/pdb1F16/pdb], PDB 2K7W [https://doi. org/10.2210/pdb2K7W/pdb]. Source data are provided with this paper.

Received: 9 December 2020; Accepted: 14 January 2021;

Published online: 18 February 2021

\section{References}

1. Singh, R., Letai, A. \& Sarosiek, K. Regulation of apoptosis in health and disease: the balancing act of BCL-2 family proteins. Nat. Rev. Mol. Cell Biol. 20, 175-193 (2019).

2. Delbridge, A. et al. Thirty years of BCL-2: translating cell death discoveries into novel cancer therapies. Nat. Rev. Cancer 16, 99-109 (2016).

3. Del Re, D. P., Amgalan, D., Linkermann, A., Liu, Q. \& Kitsis, R. N. Fundamental mechanisms of regulated cell death and implications for heart disease. Physiol. Rev. 99, 1765-1817 (2019).

4. Hollville, E., Romero, S. E. \& Deshmukh, M. Apoptotic cell death regulation in neurons. FEBS J. 286, 3276-3298 (2019).

5. Luna-Vargas, M. P. \& Chipuk, J. E. The deadly landscape of pro-apoptotic BCL-2 proteins in the outer mitochondrial membrane. Febs. J. 283, 2676-2689 (2016).

6. Tait, S. W. \& Green, D. R. Mitochondria and cell death: outer membrane permeabilization and beyond. Nat. Rev. Mol. Cell Biol. 11, 621-632 (2010)

7. Kale, J., Osterlund, E. \& Andrews, D. BCL-2 family proteins: changing partners in the dance towards death. Cell Death Differ. 25, 65-80 (2018).

8. Huska, J. D., Lamb, H. M. \& Hardwick, J. M. Overview of BCL-2 family proteins and therapeutic potentials. Methods Mol. Biol. 1877, 1-21 (2019).

9. Edlich, F. et al. Bcl- $x_{\mathrm{L}}$ retrotranslocates Bax from the mitochondria into the cytosol. Cell 145, 104-116 (2011).

10. Garner, T. P. et al. An autoinhibited dimeric form of BAX regulates the BAX activation pathway. Mol. Cell 63, 485-497 (2016).

11. Moldoveanu, T. et al. The X-ray structure of a BAK homodimer reveals an inhibitory zinc binding site. Mol. Cell 24, 677-688 (2006).

12. Sattler, M. et al. Structure of Bcl-xL-Bak peptide complex: recognition between regulators of apoptosis. Science 275, 983-986 (1997). 983.

13. Chen, H. C. et al. An interconnected hierarchical model of cell death regulation by the BCL-2 family. Nat. Cell Biol. 17, 1270-1281 (2015).

14. Ren, D. et al. BID, BIM, and PUMA are essential for activation of the BAX- and BAK-dependent cell death program. Science 330, 1390-1393 (2010).

15. Chen, L. et al. Differential targeting of prosurvival Bcl-2 proteins by their $\mathrm{BH} 3-$ only ligands allows complementary apoptotic function. Mol. Cell 17, 393-403 (2005)

16. Suzuki, M., Youle, R. J. \& Tjandra, N. Structure of Bax: coregulation of dimer formation and intracellular localization. Cell 103, 645-654 (2000).

17. Gavathiotis, E. et al. BAX activation is initiated at a novel interaction site. Nature 455, 1076-1081 (2008).

18. Gavathiotis, E. et al. BH3-triggered structural re-organization drives the activation of pro-apoptotic BAX. Mol. Cell 40, 481-492 (2010).

19. Kim, H. et al. Stepwise activation of BAX and BAK by tBID, BIM, and PUMA initiates mitochondrial apoptosis. Mol. Cell 36, 487-499 (2009).

20. Czabotar, P. E. et al. Bax crystal structures reveal how $\mathrm{BH} 3$ domains activate bax and nucleat its oligomerization to induce apoptosis. Cell 152, 519-531 (2013).

21. Bleicken, S. et al. Structural model of active Bax at the membrane. Mol. Cell 56, 496-505 (2014).

22. Zhang, Z. et al. BH3-in-groove dimerization initiates and helix 9 dimerization expands Bax pore assembly in membranes. EMBO J. 35, 208-236 (2016).

23. Fuchs, Y. \& Steller, H. Programmed cell death in animal development and disease. Cell 147, 742-758 (2011)

24. Perez, G. I. et al. Prolongation of ovarian lifespan into advanced chronological age by Bax-deficiency. Nat. Genet. 21, 200-203 (1999).

25. Libby, R. T. et al. Susceptibility to neurodegeneration in a glaucoma is modified by Bax gene dosage. PLoS. Genet. 1, 17-26 (2005).

26. Ben-Ari, Z. et al. Bax ablation protects against hepatic ischemia/reperfusion injury in transgenic mice. Liver Transpl. 13, 1181-1188 (2007).

27. Hochhauser, E. et al. Bax deficiency reduces infarct size and improves longterm function after myocardial infarction. Cell Biochem. Biophys. 47, 11-20 (2007).

28. Garner, T. P., Lopez, A., Reyna, D. E., Spitz, A. Z. \& Gavathiotis, E. Progress in targeting the BCL-2 family of proteins. Curr. Opin. Chem. Biol. 39, 133-142 (2017).

29. Walensky, L. D. Targeting BAX to drug death directly. Nat. Chem. Biol. 15, 657-665 (2019).

30. Garner, T. P. et al. Small-molecule allosteric inhibitors of BAX. Nat. Chem. Biol. 15, 322-330 (2019).

31. Amgalan, D. et al. Small molecule allosteric inhibition of BAX protects against doxorubicin-induced cardiomyopathy. Nat. Cancer 1, 315-328 (2020).

32. Pushpakom, S. et al. Drug repurposing: progress, challenges and recommendations. Nat. Rev. Drug Discov. 18, 41-58 (2019).

33. Gavathiotis, E. et al. Direct and selective small-molecule activation of proapoptotic BAX. Nat. Chem. Biol. 8, 639-645 (2012). 
34. Reyna, D. E. et al. Direct activation of BAX by BTSA1 overcomes apoptosis resistance in acute myeloid leukemia. Cancer Cell 32, 490-505 (2017).

35. Zhang, Y. \& Kolesar, J. M. Eltrombopag: anoral thrombopoietin receptor agonist for the treatment of idiopathic thrombocytopenic purpura. Clin. Ther. 33, 1560-1576 (2011).

36. Vlachodimitropoulou, E. et al. Eltrombopag: a powerful chelator of cellular or extracellular iron(III) alone or combined with a second chelator. Blood 130, 1923-1933 (2017).

37. Lovell, J. F. et al. Membrane binding by tBid initiates an ordered series of events culminating in membrane permeabilization by Bax. Cell 135, 1074-1084 (2008).

38. Ma, J. et al. Structural mechanism of Bax inhibition by cytomegalovirus protein vMIA. Proc. Natl Acad. Sci. USA 109, 20901-20906 (2012).

39. Barclay, L. A. et al. Inhibition of pro-apoptotic BAX by a noncanonical interaction mechanism. Mol. Cell 57, 873-886 (2015).

40. Dengler, M. A. et al. BAX activation: mutations near its proposed noncanonical $\mathrm{BH} 3$ binding site reveal allosteric changes controlling mitochondrial association. Cell Rep. 27, 359-373 (2019).

41. Edwards, A. L. et al. Multimodal interaction with BCL-2 family proteins underlies the proapoptotic activity of PUMA BH3. Chem. Biol. 20, 888-902 (2013).

42. Erickson-Miller, C. L. et al. Preclinical activity of eltrombopag (SB-497115), an oral, nonpeptide thrombopoietin receptor agonist. Stem Cells 27, 424-430 (2009).

43. Robin, A. Y. et al. Ensemble properties of Bax determine its function. Structure 26, 1346-1359 (2018).

44. Uchime, O. et al. Synthetic antibodies inhibit Bcl-2-associated X protein (BAX) through blockade of the N-terminal activation site. J. Biol. Chem. 291, 89-102 (2016).

45. Pritz, J. et al. Allosteric sensitization of proapoptotic BAX. Nat. Chem. Biol. 13, 961-967 (2017).

46. Iyer, S. et al. Identification of an activation site in Bak and mitochondrial Bax triggered by antibodies. Nat. Commun. 7, 11734 (2016).

47. Mitchell, W. B. et al. Effect of thrombopoietin receptor agonists on the apoptotic profile of platelets in patients with chronic immune thrombocytopenia. Am. J. Hematol. 89, E228-E234 (2014).

48. Marintchev, A., Frueh, D. \& Wagner, G. NMR methods for studying proteinprotein interactions involved in translation initiation. Methods Enzymol. 430, 283-331 (2007).

49. Deschamps, M. L., Pilka, E. S., Potts, J. R., Campbell, I. D. \& Boyd, J. Probing protein-peptide binding surfaces using charged stable free radicals and transverse paramagnetic relaxation enhancement (PRE). J. Biomol. NMR 31, 155-160 (2005).

50. Morin, A. et al. Collaboration gets the most out of software. eLife 2, e01456 (2013).

51. Greenidge, P. A., Kramer, C., Mozziconacci, J. C. \& Wolf, R. M. MM/ GBSA binding energy prediction on the PDBbind data set: successes, failures, and directions for further improvement. J. Chem. Inf. Model 53, 201-209 (2013).

\section{Acknowledgements}

Studies were supported by the Irma T. Hirschl Trust Career Award to E.G. A.Z.S. was supported by NIH F30CA228453 fellowship and T32GM007288 training grant. E.G. is supported from NIH awards R01CA178394 and P01AG031782, and NMR resources are supported from NIH awards 1S10OD016305 and P30 CA013330.

\section{Author contributions}

A.Z.S. performed biochemical, NMR, molecular modeling and cell-based studies. E.Z. performed cell-based studies, D.E.R. assisted with cell-based studies, T.P.G. assisted with NMR studies. E.G. conceived the study, designed experiments, wrote the paper with A.Z.S., which was edited by all authors.

\section{Competing interests}

E.G. is a founder and scientific advisor of BAKX Therapeutics, Stelexis Therapeutics, Selphagy Therapeutics, and scientific advisor to Life Biosciences. None of the above companies have sponsored this research. The remaining authors declare no competing interests.

\section{Additional information}

Supplementary information The online version contains supplementary material available at https://doi.org/10.1038/s41467-021-21224-1.

Correspondence and requests for materials should be addressed to E.G.

Peer review information Nature Communications thanks the anonymous reviewers for their contribution to the peer review of this work. Peer reviewer reports are available.

Reprints and permission information is available at http://www.nature.com/reprints Publisher's note Springer Nature remains neutral with regard to jurisdictional claims in published maps and institutional affiliations.

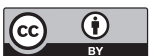

Open Access This article is licensed under a Creative Commons Attribution 4.0 International License, which permits use, sharing, adaptation, distribution and reproduction in any medium or format, as long as you give appropriate credit to the original author(s) and the source, provide a link to the Creative Commons license, and indicate if changes were made. The images or other third party material in this article are included in the article's Creative Commons license, unless indicated otherwise in a credit line to the material. If material is not included in the article's Creative Commons license and your intended use is not permitted by statutory regulation or exceeds the permitted use, you will need to obtain permission directly from the copyright holder. To view a copy of this license, visit http://creativecommons.org/ licenses/by/4.0/.

(c) The Author(s) 2021 